\title{
Bases of the quantum matrix bialgebra and induced sign characters of the Hecke algebra
}

\author{
Ryan Kaliszewski ${ }^{1}$. Justin Lambright ${ }^{2} \cdot$ Mark Skandera $^{1}$
}

Received: 25 February 2018 / Accepted: 19 June 2018 / Published online: 28 June 2018

(c) Springer Science+Business Media, LLC, part of Springer Nature 2018

\section{Abstract}

We combinatorially describe entries of the transition matrices which relate monomial bases of the zero-weight space of the quantum matrix bialgebra. This description leads to a combinatorial rule for evaluating induced sign characters of the type $A$ Hecke algebra $H_{n}(q)$ at all elements of the form $\left(1+T_{s_{i_{1}}}\right) \cdots\left(1+T_{s_{i_{m}}}\right)$, including the Kazhdan-Lusztig basis elements indexed by 321-hexagon-avoiding permutations. This result is the first subtraction-free rule for evaluating all elements of a basis of the $H_{n}(q)$-trace space at all elements of a basis of $H_{n}(q)$.

\section{Introduction}

Define the symmetric group algebra $\mathbb{Z}\left[\mathfrak{S}_{n}\right]$ and the (type A Iwahori-) Hecke algebra $H_{n}(q)$ to be the algebras with multiplicative identity elements $e$ and $T_{e}$, respectively, generated over $\mathbb{Z}$ and $\mathbb{Z}\left[q^{\frac{1}{2}}, q^{-\frac{1}{2}}\right]$ by elements $s_{1}, \ldots, s_{n-1}$ and $T_{s_{1}}, \ldots, T_{s_{n-1}}$, subject to the relations

$$
\begin{array}{lll}
s_{i}^{2}=e & T_{s_{i}}^{2}=(q-1) T_{s_{i}}+q T_{e} & \text { for } i=1, \ldots, n-1, \\
s_{i} s_{j} s_{i}=s_{j} s_{i} s_{j} & T_{s_{i}} T_{s_{j}} T_{s_{i}}=T_{s_{j}} T_{s_{i}} T_{s_{j}} & \text { for }|i-j|=1, \\
s_{i} s_{j}=s_{j} s_{i} & T_{s_{i}} T_{s_{j}}=T_{s_{j}} T_{s_{i}} & \text { for }|i-j| \geq 2 .
\end{array}
$$

Mark Skandera

mas906@lehigh.edu

Ryan Kaliszewski

ryk216@lehigh.edu

Justin Lambright

jjlambright@anderson.edu

1 Department of Mathematics, Lehigh University, Bethlehem, PA, USA

2 School of Science and Engineering, Anderson University, Anderson, IN, USA 
Analogous to the natural basis $\left\{w \mid w \in \mathfrak{S}_{n}\right\}$ of $\mathbb{Z}\left[\mathfrak{S}_{n}\right]$ is the natural basis $\left\{T_{w} \mid w \in\right.$ $\left.\mathfrak{S}_{n}\right\}$ of $H_{n}(q)$, where we define $T_{w}=T_{s_{i_{1}}} \cdots T_{s_{i_{\ell}}}$ whenever $s_{i_{1}} \cdots s_{i_{\ell}}$ is a reduced (short as possible) expression for $w$ in $\mathfrak{S}_{n}$. We call $\ell$ the length of $w$ and write $\ell=\ell(w)$. We define the one-line notation $w_{1} \cdots w_{n}$ of $w \in \mathfrak{S}_{n}$ by letting any expression for $w$ act on the word $1 \cdots n$, where each generator $s_{j}$ acts on an $n$-letter word by swapping the letters in positions $j$ and $j+1$,

$$
s_{j} \circ v_{1} \cdots v_{n}=v_{1} \cdots v_{j-1} v_{j+1} v_{j} v_{j+2} \cdots v_{n} .
$$

It is known that $\ell(w)$ is equal to $\operatorname{INV}(w)$, the number of inversions in the one-line notation $w_{1} \cdots w_{n}$ of $w$. The specialization of $H_{n}(q)$ at $q^{\frac{1}{2}}=1$ is isomorphic to $\mathbb{Z}\left[\mathfrak{S}_{n}\right]$. Two partial orders arising in the study of $\mathfrak{S}_{n}$ are the Bruhat order $\leq$ and the weak order $\leq_{W}$ defined by

$u \leq v$ if every reduced expression for $v$ contains a reduced expression for $u$,

$u \leq_{W} v$ if some reduced expression for vends with a reduced expression for $u$.

In addition to the natural bases of $\mathbb{Z}\left[\mathfrak{S}_{n}\right]$ and $H_{n}(q)$, we have the (signless) Kazhdan-Lusztig bases [8] $\left\{C_{w}^{\prime}(1) \mid w \in \mathfrak{S}_{n}\right\},\left\{C_{w}^{\prime}(q) \mid w \in \mathfrak{S}_{n}\right\}$, defined in terms of certain Kazhdan-Lusztig polynomials $\left\{P_{v, w}(q) \mid v, w \in \mathfrak{S}_{n}\right\}$ in $\mathbb{N}[q]$ by

$$
C_{w}^{\prime}(1)=\sum_{v \leq w} P_{v, w}(1) v, \quad C_{w}^{\prime}(q)=q_{w}^{-1} \sum_{v \leq w} P_{v, w}(q) T_{v},
$$

where we define $q_{w}=q^{\frac{\ell(w)}{2}}$. A modification $\left\{q_{w} C_{w}^{\prime}(q) \mid w \in \mathfrak{S}_{n}\right\}$ of the second basis belongs to $\operatorname{span}_{\mathbb{N}[q]}\left\{T_{w} \mid w \in \mathfrak{S}_{n}\right\}$.

Representations of $\mathbb{Z}\left[\mathfrak{S}_{n}\right]$ and $H_{n}(q)$ are often studied in terms of $\mathbb{Z}$ - and $\mathbb{Z}\left[q^{\frac{1}{2}}, q^{-\frac{1}{2}}\right]$-linear functionals called characters. The $\mathbb{Z}$-span of the $\mathfrak{S}_{n}$-characters is called the space of $\mathfrak{S}_{n}$-class functions and has dimension equal to the number of integer partitions of $n$. Two well-studied bases are the irreducible characters $\left\{\chi^{\lambda} \mid \lambda \vdash n\right\}$, and induced sign characters $\left\{\epsilon^{\lambda} \mid \lambda \vdash n\right\}$, where $\lambda \vdash n$ denotes that $\lambda$ is a partition of $n$. The $\mathbb{Z}\left[q^{\frac{1}{2}}, q^{-\frac{1}{2}}\right]$-span of the $H_{n}(q)$-characters, called the space of $H_{n}(q)$-traces, has the same dimension and analogous character bases $\left\{\chi_{q}^{\lambda} \mid \lambda \vdash n\right\},\left\{\epsilon_{q}^{\lambda} \mid \lambda \vdash n\right\}$, specializing at $q^{\frac{1}{2}}=1$ to the $\mathfrak{S}_{n}$-character bases. In each space, the Kostka numbers $\left\{K_{\lambda, \mu} \mid \lambda, \mu \vdash n\right\} \subseteq \mathbb{N}$ describe the expansion of induced sign characters in the irreducible character basis, just as they describe the expansion of elementary symmetric functions in the Schur basis of the space of homogeneous degree $n$ symmetric functions,

$$
\epsilon^{\lambda}=\sum_{\mu \vdash n} K_{\mu^{\top}, \lambda} \chi^{\mu}, \quad \epsilon_{q}^{\lambda}=\sum_{\mu \vdash n} K_{\mu^{\top}, \lambda} \chi_{q}^{\mu}, \quad e_{\lambda}=\sum_{\mu \vdash n} K_{\mu^{\top}, \lambda} s_{\mu} .
$$

(see, e.g., [13]). Here $\mu^{\top}$ denotes the transpose or conjugate of the partition $\mu$. 
The above characters $\theta \in\left\{\chi^{\lambda}, \epsilon^{\lambda}\right\}$ of $\mathfrak{S}_{n}$ satisfy $\theta^{\lambda}(z) \in \mathbb{Z}$ for all $z \in \mathbb{Z}\left[\mathfrak{S}_{n}\right]$ and $\lambda \vdash n$. An ideal combinatorial formula for such evaluations would define sets $R, S$ so that we have $\theta(z)=(-1)^{|S|}|R|$, or simply $\theta(z)=|R|$ if $\theta(z) \in \mathbb{N}$. For $z$ in the natural or Kazhdan-Lusztig basis of $\mathbb{Z}\left[\mathfrak{S}_{n}\right]$ we have the following results and open problems.

\begin{tabular}{lllll}
\hline & Do we have & Can we interpret & Do we have & Can we interpret \\
$\theta$ & $\theta(w) \in \mathbb{N}$ & $\theta(w)$ as $(-1)^{|S|}|R|$ & $\theta\left(C_{w}^{\prime}(1)\right) \in \mathbb{N}$ & $\theta\left(C_{w}^{\prime}(1)\right)$ as $|R|$ \\
& for all $w \in \mathfrak{S}_{n} ?$ & for all $w \in \mathfrak{S}_{n} ?$ & for all $w \in \mathfrak{S}_{n} ?$ & for all $w \in \mathfrak{S}_{n} ?$ \\
\hline$\epsilon^{\lambda}$ & No & Yes & Yes & Open \\
$\chi^{\lambda}$ & No & Open & Yes & Open \\
\hline
\end{tabular}

Of the four combinatorial interpretations asked for in the above table, the only one which is known may be described as follows. Let $\lambda=\left(\lambda_{1}, \ldots, \lambda_{r}\right)$. Then we have

$$
\epsilon^{\lambda}(w)=(-1)^{|S(w)|}|R(w, \lambda)|
$$

where $S(w)$ is the set of inversions in the one-line notation of $w$ and $R(w, \lambda)$ is the set of labelings of the cycles of $w$ by $1, \ldots, r$ such that exactly $\lambda_{i}$ letters are contained in the cycles labeled $i$ (cf. formula for $M(f, p)_{\lambda, \mu}$ in [1, p. 9]). For example, consider the partition $\lambda=(5,4) \vdash 9$ and permutation $w=234167589 \in \mathfrak{S}_{9}$ with $\operatorname{INV}(w)=5$. Writing $w$ in cycle notation as $(1,2,3,4)(5,6,7)(8)(9)$, we may label the cycles in three ways so that $\lambda_{1}=5$ letters belong to cycles labeled 1 and $\lambda_{2}=4$ letters belong to cycles labeled 2 :

$\begin{array}{cccc}\frac{(1,2,3,4)}{1} \frac{(5,6,7)}{2} & \frac{(8)}{1} & \frac{(9)}{2,} \\ 1 & 2 & 2 & 1, \\ 2 & 1 & 1 & 1 .\end{array}$

Thus we have $\epsilon^{\lambda}(w)=(-1)^{5} 3=-3$.

The number $\chi^{\lambda}(w)$ may be computed by the well-known Murnaghan-Nakayama algorithm but has no conjectured expression of the type stated above (see, e.g., [13]). Interpretations of $\epsilon^{\lambda}\left(C_{w}^{\prime}(1)\right)$ and $\chi^{\lambda}\left(C_{w}^{\prime}(1)\right)$ are not known for general $w \in \mathfrak{S}_{n}$, but nonnegativity follows from work of Haiman [6] and Stembridge [15]. In the special case that $w$ avoids the patterns 3412 and 4231, interpretations of these numbers are given in [4, Thm.4.7]. We say that $w \in \mathfrak{S}_{n}$ avoids the pattern $p_{1} \cdots p_{k} \in \mathfrak{S}_{k}$ if no subsequence $\left(w_{i_{1}}, \ldots, w_{i_{k}}\right)$ of $w_{1} \cdots w_{n}$ consists of letters appearing in the same relative order as $p_{1} \cdots p_{k}$.

The characters $\theta_{q} \in\left\{\chi_{q}^{\lambda}, \epsilon_{q}^{\lambda}\right\}$ of $H_{n}(q)$ satisfy $\theta_{q}(z) \in \mathbb{Z}[q]$ for all $z \in H_{n}(q)$ and $\lambda \vdash n$. An ideal combinatorial formula for such evaluations would define sequences $\left(S_{k}\right)_{k \geq 0},\left(R_{k}\right)_{k \geq 0}$ of sets so that we have $\theta_{q}(z)=\sum_{k}(-1)^{\left|S_{k}\right|}\left|R_{k}\right| q^{k}$, or simply $\theta_{q}(z)=$ 
$\sum_{k}\left|R_{k}\right| q^{k}$ if $\theta_{q}(z) \in \mathbb{N}[q]$. For $z$ in the natural basis or modified Kazhdan-Lusztig basis of $H_{n}(q)$ we have the following results and open problems.

\begin{tabular}{|c|c|c|c|c|}
\hline$\theta_{q}$ & $\begin{array}{l}\text { Do we have } \\
\begin{array}{l}\theta_{q}\left(T_{w}\right) \in \mathbb{N}[q] \\
\text { for all } w \in \mathfrak{S}_{n} ?\end{array}\end{array}$ & $\begin{array}{l}\text { Can we interpret } \\
\qquad \theta_{q}\left(T_{w}\right) \text { as } \\
\sum_{k}(-1)^{\left|S_{k}\right|}\left|R_{k}\right| q^{k} \\
\text { for all } w \in \mathfrak{S}_{n} ?\end{array}$ & $\begin{array}{l}\text { Do we have } \\
\theta_{q}\left(q_{w} C_{w}^{\prime}(q)\right) \quad \in \mathbb{N}[q] \\
\quad \text { for all } w \in \mathfrak{S}_{n} ?\end{array}$ & $\begin{array}{l}\text { Can we interpret } \\
\theta_{q}\left(q w C_{w}^{\prime}(q)\right) \text { as } \\
\qquad \sum_{k}\left|R_{k}\right| q^{k} \\
\text { for all } w \in \mathfrak{S}_{n} \text { ? }\end{array}$ \\
\hline$\epsilon_{q}^{\lambda}$ & No & Open & Yes & Open \\
\hline$\chi_{q}^{\lambda}$ & No & Open & Yes & Open \\
\hline
\end{tabular}

The polynomial $\chi_{q}^{\lambda}\left(T_{w}\right)$, and therefore $\epsilon_{q}^{\lambda}\left(T_{w}\right)$, may be computed via a $q$-extension of the Murnaghan-Nakayama algorithm (see, e.g., [11]). However, neither of these has a conjectured expression of the type asked for above. Interpretations of $\epsilon_{q}^{\lambda}\left(q_{w} C_{w}^{\prime}(q)\right)$ and $\chi_{q}^{\lambda}\left(q_{w} C_{w}^{\prime}(q)\right)$ are not known for general $w \in \mathfrak{S}_{n}$, but results concerning containment in $\mathbb{N}[q]$ follow from work of Haiman [6]. In the special case that $w$ avoids the patterns 3412 and 4231, formulas for these polynomials are given in [4, Thms. 6.4, 8.1].

To obtain ideal combinatorial interpretations analogous to those asked for above, we will consider the infinite spanning set of $H_{n}(q)$ which consists of all elements of the form

$$
\left(1+T_{s_{i_{1}}}\right) \cdots\left(1+T_{s_{i_{m}}}\right)=q^{\frac{m}{2}} C_{s_{i_{1}}}^{\prime}(q) \cdots C_{s_{i_{m}}}^{\prime}(q),
$$

where $s_{i_{1}} \cdots s_{i_{m}}$ varies over all products of generators of $\mathfrak{S}_{n}$. It is easy to see that if we arbitrarily choose one reduced expression for each element of $\mathfrak{S}_{n}$, then the $n$ ! corresponding products (1.4) form a basis for $H_{n}(q)$. Different collections of reduced expressions can yield different bases. For $z$ belonging to the above spanning set, we have the following results and open problems.

\begin{tabular}{|c|c|c|}
\hline$\theta_{q}$ & $\begin{array}{c}\text { Do we have } \\
\theta_{q}\left(\left(1+T_{s_{i_{1}}}\right) \cdots\left(1+T_{s_{i_{m}}}\right)\right) \quad \in \mathbb{N}[q] \\
\text { for all } s_{i_{1}} \cdots s_{i_{m}} ?\end{array}$ & $\begin{array}{c}\text { Can we interpret } \\
\theta_{q}\left(\left(1+T_{s_{i_{1}}}\right) \cdots\left(1+T_{s_{i_{m}}}\right)\right) \text { as } \\
\sum_{k}\left|R_{k}\right| q^{k} \text { for all } s_{i_{1}} \cdots s_{i_{m}} ?\end{array}$ \\
\hline $\begin{array}{l}\epsilon_{q}^{\lambda} \\
\chi_{q}^{\lambda}\end{array}$ & $\begin{array}{l}\text { Yes } \\
\text { Yes }\end{array}$ & $\begin{array}{l}\text { Stated in Sect. } 5 \\
\text { Open }\end{array}$ \\
\hline
\end{tabular}

Results concerning containment in $\mathbb{N}[q]$ follow from work of Haiman, since every product of the form $q_{u} C_{u}^{\prime}(q) q_{v} C_{v}^{\prime}(q)$ belongs to $\operatorname{span}_{\mathbb{N}[q]}\left\{q_{w} C_{w}^{\prime}(q) \mid w \in \mathfrak{S}_{n}\right\}$ (see [6, Appendix]). Interpretation of the polynomials $\epsilon_{q}^{\lambda}\left(\left(1+T_{s_{i_{1}}}\right) \cdots\left(1+T_{s_{i_{m}}}\right)\right)$ is new and is the first result of its kind to include evaluation of all elements of a basis of the $H_{n}(q)$ trace space at all elements of a basis of $H_{n}(q)$. Its justification depends upon the 
transition matrices which relate natural bases of Drinfeld's quantum matrix bialgebra, and an identity in this bialgebra which was stated by Konvalinka and the third author [9, Thm. 5.4].

In Sect. 2 we introduce the quantum matrix bialgebra $\mathcal{A}$ and prove combinatorial formulas for the entries of transition matrices that relate monomial bases of the zeroweight space of $\mathcal{A}$. In Sect. 3 we define a function $\sigma: \mathcal{A} \rightarrow \mathbb{Z}\left[q^{\frac{1}{2}}, q^{-\frac{1}{2}}\right]$ which allows us to compute $\theta_{q}\left(\left(1+T_{s_{i_{1}}}\right) \cdots\left(1+T_{s_{i_{m}}}\right)\right)$ for any linear function $\theta_{q}: H_{n}(q) \rightarrow$ $\mathbb{Z}\left[q^{\frac{1}{2}}, q^{-\frac{1}{2}}\right]$ in terms of a generating function in $\mathcal{A}$ for $\theta_{q}$ and a wiring diagram for the product $s_{i_{1}} \cdots s_{i_{m}}$. In Sects. 4 and 5 we use the map $\sigma$ to combinatorially evaluate induced sign characters of $H_{n}(q)$ at all elements of the spanning set (1.4). We finish with some open problems in Sect. 6.

\section{Bases of the zero-weight space of the quantum matrix bialgebra}

The study of quantum groups in the 1980s led to the study of algebras of functions on these and to the related quantum matrix bialgebra $\mathcal{A}=\mathcal{A}(n, q) . \mathcal{A}$ is the associative algebra with unit 1 generated over $\mathbb{Z}\left[q^{\frac{1}{2}}, q^{-\frac{1}{2}}\right]$ by $n^{2}$ variables $x=\left(x_{1,1}, \ldots, x_{n, n}\right)$, subject to the relations

$$
\begin{aligned}
& x_{i, \ell} x_{i, k}=q^{\frac{1}{2}} x_{i, k} x_{i, \ell}, \quad x_{j, k} x_{i, \ell}=x_{i, \ell} x_{j, k}, \\
& x_{j, k} x_{i, k}=q^{\frac{1}{2}} x_{i, k} x_{j, k}, \quad x_{j, \ell} x_{i, k}=x_{i, k} x_{j, \ell}+\left(q^{\frac{1}{2}}-q^{-\frac{1}{2}}\right) x_{i, \ell} x_{j, k},
\end{aligned}
$$

for all indices $1 \leq i<j \leq n$ and $1 \leq k<\ell \leq n$. The counit and coproduct maps

$$
\varepsilon\left(x_{i, j}\right)=\delta_{i, j}, \quad \Delta\left(x_{i, j}\right)=\sum_{k=1}^{n} x_{i, k} \otimes x_{k, j}
$$

give $\mathcal{A}$ a bialgebra structure. While $\mathcal{A}$ is not a Hopf algebra, two Hopf algebras closely related to it are the quantum coordinate rings of $\mathrm{SL}_{n}(\mathbb{C})$ and $\mathrm{GL}_{n}(\mathbb{C})$,

$$
\mathcal{O}_{q}\left(\mathrm{SL}_{n}(\mathbb{C})\right) \cong \mathbb{C} \otimes \mathcal{A} /\left(\operatorname{det}_{q}(x)-1\right), \quad \mathcal{O}_{q}\left(\mathrm{GL}_{n}(\mathbb{C})\right) \cong \mathbb{C} \otimes \mathcal{A}[t] /\left(\operatorname{det}_{q}(x) t-1\right),
$$

where

$$
\operatorname{det}_{q}(x) \underset{\operatorname{def}}{=} \sum_{v \in \mathfrak{S}_{n}}\left(-q^{-\frac{1}{2}}\right)^{\ell(v)} x_{1, v_{1}} \cdots x_{n, v_{n}}=\sum_{v \in \mathfrak{S}_{n}}\left(-q^{-\frac{1}{2}}\right)^{\ell(v)} x_{v_{1}, 1} \cdots x_{v_{n}, n}
$$

is the $(n \times n)$ quantum determinant of the matrix $x=\left(x_{i, j}\right)$. (The second equality holds in $\mathcal{A}$ but not in the noncommutative ring $\mathbb{Z}\left[q^{\frac{1}{2}}, q^{-\frac{1}{2}}\right]\left\langle x_{1,1}, \ldots, x_{n, n}\right\rangle$.) The antipode maps of these Hopf algebras are

$$
\mathcal{S}\left(x_{i, j}\right)=\left(-q^{\frac{1}{2}}\right)^{j-i} \operatorname{det}_{q}\left(x_{[n] \backslash\{j\},[n] \backslash\{i\}}\right), \quad \mathcal{S}\left(x_{i, j}\right)=\frac{\left(-q^{\frac{1}{2}}\right)^{j-i} \operatorname{det}_{q}\left(x_{[n] \backslash\{j\},[n] \backslash\{i\}}\right)}{\operatorname{det}_{q}(x)},
$$


respectively, where

$$
[n] \underset{\mathrm{def}}{=}\{1, \ldots, n\}, \quad x_{L, M} \underset{\mathrm{def}}{=}\left(x_{\ell, m}\right)_{\ell \in L, m \in M},
$$

and $\operatorname{det}_{q}\left(x_{L, M}\right)$ is defined analogously to (2.2), assuming $|L|=|M|$. Specializing $\mathcal{A}$ at $q^{\frac{1}{2}}=1$, we obtain the commutative ring $\mathbb{Z}\left[x_{1,1}, \ldots, x_{n, n}\right]$.

$\mathcal{A}$ has a natural $\mathbb{Z}\left[q^{\frac{1}{2}}, q^{-\frac{1}{2}}\right]$-basis $\left\{x_{1,1}^{a_{1,1}} \cdots x_{n, n}^{a_{n, n}} \mid a_{1,1}, \ldots, a_{n, n} \in \mathbb{N}\right\}$ of monomials in which variables appear in lexicographic order, and the relations (2.1) provide an algorithm for expressing any other monomial in terms of this basis (see, e.g., [17, Lem.2.1]). The submodule $\mathcal{A}_{[n],[n]}$ spanned by the monomials

$$
\left\{x^{u, v} \underset{\mathrm{def}}{=} x_{u_{1}, v_{1}} \cdots x_{u_{n}, v_{n}} \mid u, v \in \mathfrak{S}_{n}\right\}
$$

is called the zero-weight space of $\mathcal{A}$ and has the natural basis $\left\{x^{e, w} \mid w \in \mathfrak{S}_{n}\right\}$. The relations (2.1) imply that the monomials $\left\{x^{u, v} \mid u, v \in \mathfrak{S}_{n}\right\}$ satisfy

$$
x^{u, v}= \begin{cases}x^{s_{i} u, s_{i} v} & \text { if } s_{i} u<u \text { and } s_{i} v>v \\ x^{s_{i} u, s_{i} v}+\left(q^{\frac{1}{2}}-q^{-\frac{1}{2}}\right) x^{s_{i} u, v} & \text { if } s_{i} u<u \text { and } s_{i} v<v\end{cases}
$$

It follows that for fixed $t, u, v \in \mathfrak{S}_{n}$ satisfying $t \leq_{W} u$, each monomial $x^{u, v}$ belongs to $\sum_{w} \mathbb{N}\left[q^{\frac{1}{2}}-q^{-\frac{1}{2}}\right] x^{t, w}$. In particular, since nonnegative powers of $q^{\frac{1}{2}}-q^{-\frac{1}{2}}$ are linearly independent, there are uniquely defined polynomials $\left\{r_{u, v, t, w}\left(q_{1}\right) \mid w \in \mathfrak{S}_{n}\right\}$ in $\mathbb{N}\left[q_{1}\right]$ which satisfy

$$
x^{u, v}=\sum_{w \in \mathfrak{S}_{n}} r_{u, v, t, w}\left(q^{\frac{1}{2}}-q^{-\frac{1}{2}}\right) x^{t, w} .
$$

Some of these polynomials are identically 1 or 0 .

Proposition 2.1 For fixed $t, u, v \in \mathfrak{S}_{n}$ with $t \leq_{W} u$, the polynomials $\left\{r_{u, v, t, w}\left(q_{1}\right) \mid\right.$ $\left.w \in \mathfrak{S}_{n}\right\}$ in $\mathbb{N}\left[q_{1}\right]$ satisfy $r_{u, v, t, w}\left(q_{1}\right)=0$ unless $w \geq t u^{-1} v$, and $r_{u, v, t, t u^{-1} v}\left(q_{1}\right)=1$, i.e.,

$$
x^{u, w}=x^{t, t u^{-1} w}+\sum_{v>t u^{-1} w} r_{u, w, t, v}\left(q^{\frac{1}{2}}-q^{-\frac{1}{2}}\right) x^{t, v}
$$

Proof By definition we have $r_{t, v, t, w}\left(q_{1}\right)=\delta_{v, w}$. Thus the claim holds when $u=t$. Now fix $t$ and assume that the claim holds for $\ell(u) \leq \ell(t)+k-1$, consider $u$ of length $\ell(t)+k \geq \ell(t)+1$, and let $s$ be a left descent of $u t^{-1}$ and therefore of $u$. It follows that we have $t \leq_{W} s u$.

By (2.5) and the linear independence of powers of $q^{\frac{1}{2}}-q^{-\frac{1}{2}}$, we have

$$
r_{u, v, t, w}\left(q_{1}\right)= \begin{cases}r_{s u, s v, t, w}\left(q_{1}\right) & \text { if } s v>v \\ r_{s u, s v, t, w}\left(q_{1}\right)+\left(q_{1}\right) r_{s u, v, t, w}\left(q_{1}\right) & \text { if } s v<v\end{cases}
$$


Suppose that $w \nsupseteq t u^{-1} v$. Since $t(s u)^{-1} s v=t u^{-1} v$ and $\ell(s u)=\ell(t)+k-1$, we have by induction that $r_{s u, s v, t, w}\left(q_{1}\right)=0$ in both cases of (2.7). Furthermore since $t u^{-1} s<t u^{-1}$, we have by [3, Lem. 2.2.10] that $t u^{-1} s v>t u^{-1} v$ when $s v<v$. Thus in the second case above, the condition $w \nsupseteq t u^{-1} v$ also implies that $w \nsupseteq t u^{-1} s v$, which by induction implies that $r_{s u, v, t, w}\left(q_{1}\right)=0$. Therefore we have $r_{u, v, t, w}\left(q_{1}\right)=0$ unless $w \geq t u^{-1} v$.

Setting $w=t u^{-1} v$ in (2.7) we have $r_{s u, s v, t, t u^{-1} v}\left(q_{1}\right)=1$ in both cases. When $s v<v$ we also have $r_{s u, v, t, t u^{-1} v}\left(q_{1}\right)=0$ since $t u^{-1} v \nsupseteq t u^{-1} s v$. Therefore we have $r_{u, v, t, t u^{-1} v}\left(q_{1}\right)=1$.

Corollary 2.2 For each fixed $u \in \mathfrak{S}_{n}$, the set $\left\{x^{u, v} \mid v \in \mathfrak{S}_{n}\right\}$ is a basis for $\mathcal{A}_{[n],[n] \text {. }}$.

Proof Setting $t=e$ in (2.6) and applying Proposition 2.1 we have

$$
x^{u, v}=x^{e, u^{-1} v}+\sum_{w>u^{-1} v} r_{u, v, e, u^{-1} v}\left(q^{\frac{1}{2}}-q^{-\frac{1}{2}}\right) x^{e, w} .
$$

Now ordering the monomials $x^{u, v^{(1)}}, \ldots, x^{u, v^{(n !)}}$ so that $u^{-1} v^{(1)}, \ldots, u^{-1} v^{(n !)}$ is a linear extension of the Bruhat order, we have a unitriangular system of equations.

By the unitriangularity of the coefficient matrix in the above proof, we may extend the statement containing (2.6).

Corollary 2.3 For for fixed $t, u \in \mathfrak{S}_{n}$, not necessarily related in the weak order, there are uniquely defined polynomials $\left\{r_{u, v, t, w}\left(q_{1}\right) \mid v, w \in \mathfrak{S}_{n}\right\}$ in $\mathbb{Z}\left[q_{1}\right]$ which satisfy

$$
x^{u, v}=\sum_{w \in \mathfrak{S}_{n}} r_{u, v, t, w}\left(q^{\frac{1}{2}}-q^{-\frac{1}{2}}\right) x^{t, w} .
$$

Now we turn to the problem of combinatorially interpreting coefficients of the polynomials $\left\{r_{u, v, t, w}\left(q_{1}\right) \mid u, v, t, w \in \mathfrak{S}_{n}\right\}$ when $t \leq_{W} u$. To begin, we consider a seemingly unrelated generating function for certain walks in the weak order.

Definition 2.4 Fix permutations $t, u, v, w \in \mathfrak{S}_{n}$ with $t \leq_{W} u$, and a reduced expression $s_{i_{1}} \cdots s_{i_{k}}$ for $u t^{-1}$. Define $C_{u, v, t, w}^{b}\left(s_{i_{1}} \cdots s_{i_{k}}\right)$ to be the set of sequences $\pi=\left(\pi^{(0)}, \ldots, \pi^{(k)}\right)$ satisfying

(1) $\pi^{(0)}=v, \pi^{(k)}=w$,

(2) $\pi^{(j)} \in\left\{s_{i_{j}} \pi^{(j-1)}, \pi^{(j-1)}\right\}$ for $j=1, \ldots, k$,

(3) $\pi^{(j)}=s_{i_{j}} \pi^{(j-1)}$ if $s_{i j} \pi^{(j-1)}>\pi^{(j-1)}$ for $j=1, \ldots, k$,

(4) $\pi^{(j)}=\pi^{(j-1)}$ for exactly $b$ values of $j$ for $j=1, \ldots, k$,

and define the polynomial

$$
p_{u, v, t, w}\left(q_{1} ; s_{i_{1}} \cdots s_{i_{k}}\right)=\sum_{b}\left|C_{u, v, t, w}^{b}\left(s_{i_{1}} \cdots s_{i_{k}}\right)\right| q_{1}^{b} \in \mathbb{N}\left[q_{1}\right] .
$$

Observe that we have $p_{t, v, t, w}\left(q_{1} ; \emptyset\right)=\delta_{v, w}$. We also have the following recursive formula. 
Proposition 2.5 Fix $t, u, v, w \in \mathfrak{S}_{n}$ with $t<_{W} u$, and fix a reduced expression $s_{i_{1}} \cdots s_{i_{k}}$ for $u t^{-1}$. Then we have

$$
\begin{aligned}
& p_{u, v, t, w}\left(q_{1} ; s_{i_{1}} \cdots s_{i_{k}}\right) \\
& \quad= \begin{cases}p_{s_{i_{1}} u, s_{i_{1}} v, t, w}\left(q_{1} ; s_{i_{2}} \cdots s_{i_{k}}\right) & \text { if } s_{i_{1}} v>v, \\
p_{s_{i_{1}} u, s_{i_{1}} v, t, w}\left(q_{1} ; s_{i_{2}} \cdots s_{i_{k}}\right)+q_{1} p_{s_{i_{1}} u, v, t, w}\left(q_{1} ; s_{i_{2}} \cdots s_{i_{k}}\right) & \text { if } s_{i_{1}} v<v .\end{cases}
\end{aligned}
$$

Proof The coefficient of $q_{1}^{b}$ on the left-hand side of (2.10) is $\left|C_{u, v, t, w}^{b}\left(s_{i_{1}} \cdots s_{i_{k}}\right)\right|$. Since $t<_{W} u$ and $s_{i_{1}}$ is a left descent for $u t^{-1}$, we have that $t \leq_{W} s_{i_{1}} u$ and that $s_{i_{2}} \cdots s_{i_{k}}$ is a reduced expression for $s_{i_{1}} u t^{-1}$. Thus the coefficient of $q_{1}^{b}$ on the right-hand side of (2.10) is equal to the cardinality of

$$
D \underset{\operatorname{def}}{=} \begin{cases}C_{s_{i_{1}} u, s_{i_{1}} v, t, w}^{b}\left(s_{i_{2}} \cdots s_{i_{k}}\right) & \text { if } s_{i_{1}} v>v \\ C_{s_{i_{1}} u, s_{i_{1}} v, t, w}^{b}\left(s_{i_{2}} \cdots s_{i_{k}}\right) \cup C_{s_{i_{1}} u, v, t, w}^{b-1}\left(s_{i_{2}} \cdots s_{i_{k}}\right) & \text { if } s_{i_{1}} v<v\end{cases}
$$

We claim that the map

$$
\pi=\left(\pi^{(0)}, \pi^{(1)}, \ldots, \pi^{(k)}\right) \mapsto\left(\pi^{(1)}, \ldots, \pi^{(k)}\right)
$$

is a bijection from $C_{u, v, t, w}^{b}\left(s_{i_{1}} \cdots s_{i_{k}}\right)$ to $D$. Clearly it is injective, since each element of $C_{u, v, t, w}^{b}\left(s_{i_{1}} \cdots s_{i_{k}}\right)$ satisfies $\pi^{(0)}=v$.

To see that the map (2.11) is well defined and surjective, assume first that $s_{i_{1}} v>v$. Then $\pi$ satisfies $\pi^{(1)}=s_{i_{1}} v$ and we have $b \leq k-1$. It follows that for $b=0, \ldots, k-1$, the sequence $\left(\pi^{(1)}, \ldots, \pi^{(k)}\right)$ satisfies the conditions

$\left(1^{\prime}\right) \pi^{(1)}=s_{i_{1}} v, \pi^{(k)}=w$,

(2') $\pi^{(j)} \in\left\{s_{i_{j}} \pi^{(j-1)}, \pi^{(j-1)}\right\}$ for $j=2, \ldots, k$,

(3') $\pi^{(j)}=s_{i_{j}} \pi^{(j-1)}$ if $s_{i_{j}} \pi^{(j-1)}>\pi^{(j-1)}$ for $j=2, \ldots, k$,

$\left(4^{\prime}\right) \pi^{(j)}=\pi^{(j-1)}$ for exactly $b$ values of $j$ for $j=2, \ldots, k$.

Thus $\left(\pi^{(1)}, \ldots, \pi^{(k)}\right)$ belongs to $C_{s_{i_{1}} u, s_{i_{1}} v, t, w}^{b}\left(s_{i_{2}} \cdots s_{i_{k}}\right)$. Moreover, since prepending $v$ to any sequence in $C_{s_{i_{1}} u, s_{i_{1}} v, t, w}^{b}\left(s_{i_{2}} \cdots s_{i_{k}}\right)$ produces a sequence belonging to $C_{u, v, t, w}^{b}\left(s_{i_{1}} \cdots s_{i_{k}}\right)$, the map (2.11) is surjective as well.

Now assume that $s_{i_{1}} v<v$. Then $\pi$ satisfies $\pi^{(1)}=s_{i_{1}} v$ or $\pi^{(1)}=v$. If $\pi^{(1)}=s_{i_{1}} v$, then the sequence $\left(\pi^{(1)}, \ldots, \pi^{(k)}\right)$ satisfies conditions $\left(1^{\prime}\right)-\left(4^{\prime}\right)$ above. Otherwise it satisfies conditions $\left(2^{\prime}\right)-\left(3^{\prime}\right)$ and

$\left(1^{\prime \prime}\right) \pi^{(1)}=v, \pi^{(k)}=w$,

$\left(4^{\prime \prime}\right) \pi^{(j)}=\pi^{(j-1)}$ for exactly $b-1$ values of $j$ for $j=2, \ldots, k$.

Thus the sequence $\left(\pi^{(1)}, \ldots, \pi^{(k)}\right)$ belongs to

$$
C_{s_{i_{1}} u, s_{i_{1}} v, t, w}^{b}\left(s_{i_{2}} \cdots s_{i_{k}}\right) \cup C_{s_{i_{1}} u, v, t, w}^{b-1}\left(s_{i_{2}} \cdots s_{i_{k}}\right) .
$$


Moreover, since prepending $v$ to any sequence in this union produces a sequence belonging to $C_{u, v, t, w}^{b}\left(s_{i_{1}} \cdots s_{i_{k}}\right)$, we again have surjectivity.

For fixed $t, u, w$ and reduced expression $s_{i_{1}} \cdots s_{i_{k}}$ as in Definition 2.4, the above initial conditions and recursive formula allow one to compute $\left\{p_{u, v, t, w}\left(q_{1} ; s_{i_{1}} \cdots s_{i_{k}}\right) \mid v \in\right.$ $\mathfrak{S}_{n}$ \} by considering the sets

$$
\left\{p_{s_{i_{k}} t, v, t, w}\left(q_{1} ; s_{i_{k}}\right) \mid v \in \mathfrak{S}_{n}\right\}, \quad\left\{p_{s_{i_{k-1}} s_{i_{k}} t, v, t, w}\left(q_{1} ; s_{i_{k-1}} s_{i_{k}}\right) \mid v \in \mathfrak{S}_{n}\right\}, \ldots,
$$

in order. Somewhat surprisingly, these polynomials do not depend upon the choice of a reduced expression for $u t^{-1}$, although each set $C_{u, v, t, w}^{b}\left(s_{i_{1}} \cdots s_{i_{k}}\right)$ does depend upon such a choice. Also, perhaps surprisingly, these polynomials provide a combinatorial interpretation for entries of the transition matrices relating pairs $\left(\left\{x^{u, v} \mid v \in\right.\right.$ $\left.\left.\mathfrak{S}_{n}\right\},\left\{x^{t, w} \mid w \in \mathfrak{S}_{n}\right\}\right)$ of bases of the zero-weight space of $\mathcal{A}$.

Theorem 2.6 For $t, u$ in $\mathfrak{S}_{n}$ with $t \leq_{W} u$, the polynomials $\left\{r_{u, v, t, w}\left(q_{1}\right) \mid v, w \in \mathfrak{S}_{n}\right\}$ defined in (2.6) satisfy $r_{u, v, t, w}\left(q_{1}\right)=p_{u, v, t, w}\left(q_{1}, s_{i_{1}} \cdots s_{i_{k}}\right)$, where $s_{i_{1}} \cdots s_{i_{k}}$ is any reduced expression for $u t^{-1}$.

Proof Observe that the claimed equality holds when $t=u$, since

$$
r_{u, v, u, w}\left(q_{1}\right)=p_{u, v, u, w}\left(q_{1} ; \emptyset\right)=\delta_{v, w}
$$

by (2.8) and (2.9). Now assume the equality to hold for $u$ and $t$ differing in length by at most $k-1$, and consider the case that $u$ and $t$ differ in length by $k$. Let $s$ be a left descent of $u t^{-1}$, and therefore a left descent of $u$.

Expanding both sides of (2.6) in terms of the basis $\left\{x^{t, w} \mid w \in \mathfrak{S}_{n}\right\}$ and using induction, we obtain

$$
r_{u, v, t, w}\left(q_{1}\right)= \begin{cases}p_{s u, s v, t, w}\left(q_{1} ; s_{j_{2}} \cdots s_{j_{k}}\right) & \text { if } s v>v, \\ p_{s u, s v, t, w}\left(q_{1} ; s_{j_{2}} \cdots s_{j_{k}}\right)+q_{1} p_{s u, v, t, w}\left(q_{1} ; s_{j_{2}} \cdots s_{j_{k}}\right) & \text { if } s v<v\end{cases}
$$

where $s_{j_{2}} \cdots s_{j_{k}}$ is an arbitrary reduced expression for $s u t^{-1}$. Since $s$ is an arbitrarily chosen left descent of $u t^{-1}$, we have the desired result.

Using Definition 2.4 and Theorem 2.6, we compute some special cases of the polynomials $r_{u, v, t, w}\left(q_{1}\right)$.

Proposition 2.7 Fix $w \in \mathfrak{S}_{n}$ and any generator s. We have

$$
\begin{gathered}
r_{w s, w, e, s}\left(q_{1}\right)=r_{w, w, e, e}\left(q_{1}\right)=1, \\
r_{w, w, e, s}\left(q_{1}\right)= \begin{cases}q_{1} & \text { if } w s<w, \\
0 & \text { if } w s>w .\end{cases}
\end{gathered}
$$

Proof (2.13) follows from Proposition 2.1. 
To see (2.14), consider the coefficient of $q_{1}^{b}$ in $r_{w, w, e, s}\left(q_{1}\right)$ for $b \geq 0$, and let $\ell(w)=m$. By Theorem 2.6 this is equal to the number of $m$-step walks

$$
\pi=\left(\pi^{(0)}=w, \pi^{(1)}, \ldots, \pi^{(m)}=s\right)
$$

in the weak order satisfying conditions stated in Definition 2.4. In particular, $b$ of the indices $j \in\{1, \ldots, m-1\}$ satisfy $\pi^{(j-1)}=\pi^{(j)}$, while the others satisfy $\pi^{(j-1)}>$ $\pi^{(j)}$. Since $\ell(w)-\ell(s)=m-1$, the coefficient must be 0 unless $b=1$. Furthermore, the coefficient must be 0 if $w s>w$, equivalently $s \nless_{W} w$, because in this case the shortest walk in the weak order from $w$ to $s$ consists of $m+1$ steps with no repetition.

Suppose therefore that we have $b=1$ and $w s<w$, equivalently $s<_{W} w$. Then $w$ has a reduced expression of the form $s_{i_{1}} \cdots s_{i_{m-1}} s$, and the sequence

$$
\left(w, s_{i_{1}} w, s_{i_{2}} s_{i_{1}} w, \ldots, s_{i_{m-1}} \cdots s_{i_{1}} w=s, s\right)
$$

is one walk satisfying the conditions of Definition 2.4 using the above reduced expression (which may be chosen arbitrarily by Theorem 2.6). Assume that another such walk satisfies the conditions of the definition, using the same reduced expression. Then for some index $j<m-1$ this walk satisfies $\pi^{(j)}=\pi^{(j-1)}$ and has the form

$\left(w, s_{i_{1}} w, \ldots, s_{i_{j}} \cdots s_{i_{1}} w, s_{i_{j}} \cdots s_{i_{1}} w, s_{i_{j+2}} s_{i_{j}} \cdots s_{i_{1}} w, \ldots, s s_{i_{m-1}} \cdots s_{i_{j+2}} s_{i_{j}} \cdots s_{i_{1}} w=s\right)$.

But the equation in the last component of this walk implies that we have

$$
w=s_{i_{1}} \cdots s_{i_{j}} s_{i_{j+2}} \cdots s_{i_{m-1}},
$$

contradicting the fact that $\ell(w)=m$. It follows that (2.15) is the only walk satisfying the conditions of Definition 2.4 for the chosen reduced expression, and that the coefficient of $q_{1}^{1}$ is 1 when $w s<w$.

\section{Wiring diagrams and the $q$-immanant evaluation theorem}

To evaluate induced sign characters at elements $\left(1+T_{s_{i_{1}}}\right) \cdots\left(1+T_{s_{i_{m}}}\right)$ of $H_{n}(q)$, we will associate to each such element a graph $G$ called a wiring diagram, a related matrix $B$, and a map $\sigma_{B}: \mathcal{A}_{[n],[n]} \rightarrow \mathbb{Z}\left[q^{\frac{1}{2}}, q^{-\frac{1}{2}}\right]$. A generating function $\operatorname{Imm}_{\epsilon_{q}^{\lambda}}(x) \in \mathcal{A}_{[n],[n]}$ for $\left\{\epsilon_{q}^{\lambda}\left(T_{w}\right) \mid w \in \mathfrak{S}_{n}\right\}$ will then allow us to compute

$$
\epsilon_{q}^{\lambda}\left(\left(1+T_{s_{i_{1}}}\right) \cdots\left(1+T_{s_{i_{m}}}\right)\right)=\sigma_{B}\left(\operatorname{Imm}_{\epsilon_{q}^{\lambda}}(x)\right)
$$

and to combinatorially interpret the resulting polynomial. 


\subsection{Wiring diagrams and the classical immanant evaluation identity}

Call a directed planar graph $G$ a wiring diagram if it is a concatenation of any combination of the diagrams

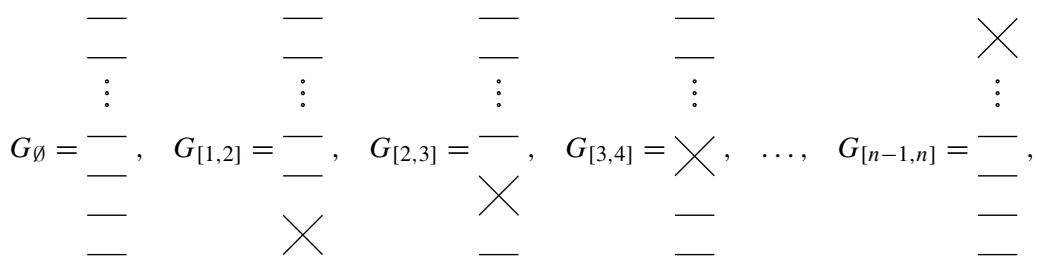

representing the elements $e, s_{1}, s_{2}, s_{3}, \ldots, s_{n-1}$ of $\mathfrak{S}_{n}$, respectively. Each wiring diagram has $n$ implicit vertices on the left and right, labeled source $1, \ldots$, source $n$ and $\operatorname{sink} 1, \ldots, \operatorname{sink} n$, respectively, from bottom to top. Edges are implicitly oriented from left to right. Let $\pi=\left(\pi_{1}, \ldots, \pi_{n}\right)$ be a sequence of source-to-sink paths in a wiring diagram $G$. We call $\pi$ a (bijective) path family if there exists a permutation $w=w_{1} \cdots w_{n} \in \mathfrak{S}_{n}$ such that $\pi_{i}$ is a path from source $i$ to sink $w_{i}$. In this case, we say more specifically that $\pi$ has type $w$. We say that the path family covers $G$ if it contains every edge exactly once.

The number of path families covering

$$
G=G_{\left[i_{1}, i_{1}+1\right]} \circ \cdots \circ G_{\left[i_{m}, i_{m}+1\right]}
$$

is $2^{m}$ : for $j=1, \ldots, m$, the two paths intersecting at the central vertex of $G_{\left[i_{j}, i_{j}+1\right]}$ either cross or do not cross at that vertex. In these two cases, we call the index $j$ a crossing or noncrossing of the path family, respectively. We call (3.3) the wiring diagram of the expression $s_{i_{1}} \cdots s_{i_{m}}$, whether or not this expression is reduced. It is well known that if we have the equality $s_{i_{1}} \cdots s_{i_{m}}=v$ in $\mathfrak{S}_{n}$, then $v$ is the type of the unique path family covering $G$ in which all indices $1, \ldots, m$ are crossings.

Alternatively, one may use the same diagram $G$ to encode the element $(1+$ $\left.s_{i_{1}}\right) \cdots\left(1+s_{i_{m}}\right)$ of $\mathbb{Z}\left[\mathfrak{S}_{n}\right]$. The $2^{m}$ terms in the expansion of this product may be written and collected as

$$
\sum_{\beta \in 2^{[m]}} s_{i_{1}}^{\beta_{1}} \cdots s_{i_{m}}^{\beta_{m}}=\sum_{v \in \mathfrak{S}_{n}} d_{v} v
$$

where the $2^{m}$ binary words $\beta=\beta_{1} \cdots \beta_{m}$ correspond to path families covering $G$ by

$$
\beta_{j}= \begin{cases}1 & \text { if } j \text { is a crossing } \\ 0 & \text { otherwise }\end{cases}
$$

and where we define $s_{i_{j}}^{0}=e$. Thus each coefficient $d_{v} \in \mathbb{N}$ in the second sum counts the number of path families of type $v$ which cover $G$. 
Similar to the above encoding is the use of $G$ to encode the element $\left(1+T_{s_{i_{1}}}\right) \cdots(1+$ $\left.T_{s_{i_{m}}}\right)$ of $H_{n}(q)$. Expanding this product and collecting terms, we have

$$
\sum_{\beta \in 2^{[m]}}\left(T_{s_{i_{1}}}\right)^{\beta_{1}} \cdots\left(T_{s_{i_{m}}}\right)^{\beta_{m}}=\sum_{v \in \mathfrak{S}_{n}} a_{v} T_{v},
$$

where binary words $\beta$ correspond to path families as in (3.4). Now the coefficients $a_{v}$ in the expansion belong to $\mathbb{N}[q]$ and are defined in terms of a path family statistic called defects. Call index $j$ a defect of path family $\pi$ if the two paths containing the central vertex of $G_{\left[i_{j}, i_{j}+1\right]}$ have previously crossed an odd number of times. (Equivalently, a crossing or noncrossing is defective if the path entering the common vertex on top has a lower source index.) We will call an index $j$ a proper crossing or noncrossing if it is not defective. Letting $\mathrm{D}(\pi)$ denote the number of defects in $\pi$ we have [5, Prop. 3.5]

$$
a_{v}=\sum_{\pi} q^{\mathrm{D}(\pi)}
$$

where the sum is over path families of type $v$ which cover $G$.

One can enhance a wiring diagram by associating to each edge a weight belonging to some ring $R$, and by defining the weight of a path to be the product of its edge weights. If $R$ is noncommutative, then one multiplies weights in the order that the corresponding edges appear in the path. For a family $\pi=\left(\pi_{1}, \ldots, \pi_{n}\right)$ of $n$ paths in a planar network, one defines $\operatorname{wgt}(\pi)=\operatorname{wgt}\left(\pi_{1}\right) \cdots \operatorname{wgt}\left(\pi_{n}\right)$. The (weighted) path matrix $B=B(G)=\left(b_{i, j}\right)$ of $G$ is defined by letting $b_{i, j}$ be the sum of weights of all paths in $G$ from source $i$ to sink $j$. Thus the product $b_{1, w_{1}} \cdots b_{n, w_{n}}$ is equal to the sum of weights of all path families of type $w$ in $G$ (covering $G$ or not). It is easy to show that path matrices respect concatenation: $B\left(G_{1} \circ G_{2}\right)=B\left(G_{1}\right) B\left(G_{2}\right)$. When $R$ is commutative, a result known as Lindström's Lemma [7,10] asserts that for row and column sets $I, J$ with $|I|=|J|$, the $\operatorname{minor} \operatorname{det}\left(B_{I, J}\right)$ is equal to the sum of weights of all nonintersecting path families from sources indexed by $I$ to sinks indexed by $J$.

Assigning weights to the edges of $G$ (3.3) can aid in the evaluation of a linear function $\theta: \mathbb{Z}\left[\mathfrak{S}_{n}\right] \rightarrow \mathbb{Z}$ at $\left(1+s_{i_{1}}\right) \cdots\left(1+s_{i_{m}}\right)$ by relating this evaluation to the generating function

$$
\operatorname{Imm}_{\theta}(x) \underset{\text { def }}{=} \sum_{w \in \mathfrak{S}_{n}} \theta(w) x_{1, w_{1}} \cdots x_{n, w_{n}} \in \mathbb{Z}\left[x_{1,1}, \ldots, x_{n, n}\right]
$$

called the $\theta$-immanant in [14, Sec.3]. In particular, for $j=1, \ldots, m$, we assign weight 1 to the $n-2$ horizontal edges of $G_{\left[i_{j}, i_{j}+1\right]}$, and we assign (commuting) indeterminate weights $z_{i_{j}, j, 1}, z_{i_{j}, j, 2}, z_{i_{j}+1, j, 1}, z_{i_{j}+1, j, 2}$ to the remaining nonhorizontal edges $a, b, c, d$, respectively,

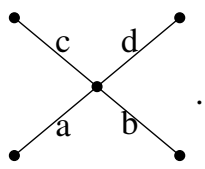


Thus wiring diagrams corresponding to expressions $s_{i_{1}} s_{i_{2}} s_{i_{3}}=s_{1} s_{2} s_{1}$ and $s_{i_{4}} s_{i_{5}} s_{i_{6}}=$ $s_{1} s_{2} s_{1}$ are weighted differently because of the different indexing of the generators. Let $z_{G}$ be the product of all $4 m$ indeterminates $z_{i, j, k}$, and for $f \in \mathbb{Z}\left[z_{1,1,1}, \ldots, z_{i_{m}, m, 2}\right]$, let $\left[z_{G}\right] f$ denote the coefficient of $z_{G}$ in $f$. Then we have the following immanant evaluation identity for wiring diagrams (cf. [16, p. 1081]).

Proposition 3.1 Assign weights to the edges of $G$ (3.3) as above and let $B$ be the resulting path matrix. Then for any linear function $\theta: \mathbb{Z}\left[\mathfrak{S}_{n}\right] \rightarrow \mathbb{Z}$ we have

$$
\theta\left(\left(1+s_{i_{1}}\right) \cdots\left(1+s_{i_{m}}\right)\right)=\left[z_{G}\right] \operatorname{Imm}_{\theta}(B) .
$$

To illustrate, we let $n=3$ and consider the element

$$
\left(1+s_{1}\right)\left(1+s_{2}\right)\left(1+s_{1}\right)=2+2 s_{1}+s_{2}+s_{1} s_{2}+s_{2} s_{1}+s_{1} s_{2} s_{1}
$$

and its wiring diagram

$$
G=G_{\left[i_{1}, i_{1}+1\right]} \circ G_{\left[i_{2}, i_{2}+1\right]} \circ G_{\left[i_{3}, i_{3}+1\right]}=G_{[1,2]} \circ G_{[2,3]} \circ G_{[1,2]}
$$

Assigning weights to the edges of $G$ we have

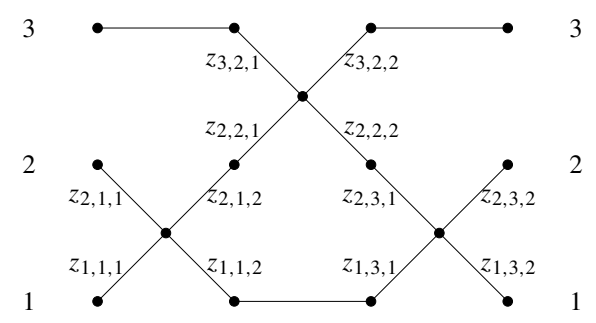

and $z_{G}=z_{1,1,1} \cdots z_{3,2,2}$. The weighted path matrix of $G$ is

$$
\begin{aligned}
& B=\left[\begin{array}{ccc}
z_{1,1,1} z_{1,1,2} & z_{1,1,1} z_{2,1,2} & 0 \\
z_{2,1,1} z_{1,1,2} & z_{2,1,1} z_{2,1,2} & 0 \\
0 & 0 & 1
\end{array}\right]\left[\begin{array}{ccc}
1 & 0 & 0 \\
0 & z_{2,2,1} z_{2,2,2} & z_{2,2,1} z_{3,2,2} \\
0 & z_{3,2,1} z_{2,2,2} & z_{3,2,1} z_{3,2,2}
\end{array}\right]\left[\begin{array}{ccc}
z_{1,3,1} z_{1,3,2} & z_{1,3,1} z_{2,3,2} & 0 \\
z_{2,3,1} z_{1,3,2} & z_{2,3,1} z_{2,3,2} & 0 \\
0 & 0 & 1
\end{array}\right] \\
& =\left[\begin{array}{ccc}
z_{1,1,1} z_{U} z_{1,3,2}+z_{1,1,1} z_{D} z_{1,3,2} & z_{1,1,1} z_{U} z_{2,3,2}+z_{1,1,1} z_{D} z_{2,3,2} & z_{1,1,1} z_{2,1,2} z_{2,2,1} z_{3,2,2} \\
z_{2,1,1} z_{U} z_{1,3,2}+z_{2,1,1} z_{D} z_{1,3,2} & z_{2,1,1} z_{U} z_{2,3,2}+z_{2,1,1} z_{D} z_{2,3,2} & z_{2,1,1} z_{2,1,2} z_{2,2,1} z_{3,2,2} \\
z_{3,2,1} z_{2,2,2} z_{2,3,1} z_{1,3,2} & z_{3,2,1} z_{2,2,2} z_{2,3,1} z_{2,3,2} & z_{3,2,1} z_{3,2,2}
\end{array}\right],
\end{aligned}
$$

where $z_{U}=z_{2,1,2} z_{2,2,1} z_{2,2,2} z_{2,3,1}, z_{D}=z_{1,1,2} z_{1,3,1}$.

Now we consider the linear function $\theta: \mathbb{Z}\left[\mathfrak{S}_{3}\right] \rightarrow \mathbb{Z}$ defined by $\theta(e)=1$, $\theta\left(s_{1} s_{2} s_{1}\right)=-1, \theta(w)=0$ otherwise. Computing the left-hand side of (3.9) we have

$$
\theta\left(\left(1+s_{1}\right)\left(1+s_{2}\right)\left(1+s_{1}\right)\right)=2-1=1 .
$$

To compute the right-hand side of (3.9), we first factor the immanant as

$$
\operatorname{Imm}_{\theta}(x)=x_{1,1} x_{2,2} x_{3,3}-x_{1,3} x_{2,2} x_{3,1}=\operatorname{det}\left(x_{13,13}\right) x_{2,2} .
$$


By Lindström's Lemma and inspection of the wiring diagram (3.11), we have

$$
\left[z_{G}\right] \operatorname{Imm}_{\theta}(B)=\left[z_{G}\right] \operatorname{det}\left(B_{13,13}\right) b_{2,2}=1,
$$

since exactly one family of paths $\pi=\left(\pi_{1}, \pi_{2}, \pi_{3}\right)$ from all sources to the corresponding sinks satisfies

(1) $\pi_{1}$ and $\pi_{3}$ do not intersect,

(2) $\pi$ covers $G$ and therefore has weight $z_{G}$.

\subsection{The $q$-immanant evaluation identity for wiring diagrams}

It is natural to ask for a $q$-analog of Proposition 3.1 which applies to the computation of $\theta_{q}\left(\left(1+T_{s_{i_{1}}}\right) \cdots\left(1+T_{s_{i_{m}}}\right)\right)$ for a linear function $\theta_{q}: H_{n}(q) \rightarrow \mathbb{Z}\left[q^{\frac{1}{2}}, q^{-\frac{1}{2}}\right]$, and which uses the generating function

$$
\operatorname{Imm}_{\theta_{q}}(x) \underset{\text { def }}{=} \sum_{w \in \mathfrak{S}_{n}} \theta_{q}\left(T_{w}\right) q_{w}^{-1} x_{1, w_{1}} \cdots x_{n, w_{n}} \in \mathcal{A}_{[n],[n]}
$$

introduced in [9, Eqn. (4.5)].

We begin by assigning weights to the edges of the wiring diagram $G$ (3.3) exactly as in (3.8). But now we define two indeterminates $z_{h, j, k}, z_{h^{\prime}, j^{\prime}, k^{\prime}}$ to commute if $j \neq j^{\prime}$ or if $k \neq k^{\prime}$; otherwise we impose the relation

$$
z_{i_{j}+1, j, k} z_{i_{j}, j, k}=q^{\frac{1}{2}} z_{i_{j}, j, k} z_{i_{j}+1, j, k}
$$

Let $Z_{G}$ be the quotient of the noncommutative ring

$$
\mathbb{Z}\left[q^{\frac{1}{2}}, q^{-\frac{1}{2}}\right]\left\langle z_{i_{j}, j, 1}, z_{i_{j}, j, 2}, z_{i_{j}+1, j, 1}, z_{i_{j}+1, j, 2} \mid j=1, \ldots, m,\right\rangle
$$

modulo the ideal generated by the above commuting and quasicommuting relations, and assume that $q^{\frac{1}{2}}, q^{-\frac{1}{2}}$ commute with all other indeterminates. Let $z_{G}$ be the product of all $4 m$ indeterminates $z_{i, j, k}$, in lexicographic order.

This small change in the indeterminates $z_{1,1,1}, \ldots, z_{i_{m}, m, 2}$ does not imply that the most naive $q$-analog of Proposition 3.1 holds, however. Indeed, the evaluation of an element of $\mathcal{A}$ at a matrix is not well defined unless the entries of that matrix satisfy the relations (2.1). We therefore define the $\mathbb{Z}\left[q^{\frac{1}{2}}, q^{-\frac{1}{2}}\right]$-linear map

$$
\begin{aligned}
\sigma_{B}: \mathcal{A}_{[n],[n]} & \rightarrow \mathbb{Z}\left[q^{\frac{1}{2}}, q^{-\frac{1}{2}}\right] \\
x_{1, v_{1}} \cdots x_{n, v_{n}} & \mapsto\left[z_{G}\right] b_{1, v_{1}} \cdots b_{n, v_{n}},
\end{aligned}
$$

where $\left[z_{G}\right] b_{1, v_{1}} \cdots b_{n, v_{n}}$ denotes the coefficient of $z_{G}$ in $b_{1, v_{1}} \cdots b_{n, v_{n}}$, taken after $b_{1, v_{1}} \cdots b_{n, v_{n}}$ is expanded in the lexicographic basis of $Z_{G}$. Note that the "substitution" $x_{i, j} \mapsto b_{i, j}$ is performed only for monomials of the form $x^{e, v}$ in $\mathcal{A}_{[n],[n]}$ : we define 
$\sigma_{B}\left(x^{u, w}\right)$ by first expanding $x^{u, w}$ in the basis $\left\{x^{e, v} \mid v \in \mathfrak{S}_{n}\right\}$, and then performing the substitution.

For example let us compute $\sigma_{B}\left(x_{2,2} x_{1,1} x_{3,3}\right)$ for the path matrix $B$ of the wiring diagram in (3.12). Using (2.1) and linearity of $\sigma_{B}$, we write

$$
\begin{aligned}
\sigma_{B}\left(x_{2,2} x_{1,1} x_{3,3}\right) & =\sigma_{B}\left(x_{1,1} x_{2,2} x_{3,3}\right)+\left(q^{\frac{1}{2}}-q^{-\frac{1}{2}}\right) \sigma_{B}\left(x_{1,2} x_{2,1} x_{3,3}\right) \\
& =\left[z_{G}\right] b_{1,1} b_{2,2} b_{3,3}+\left(q^{\frac{1}{2}}-q^{-\frac{1}{2}}\right)\left[z_{G}\right] b_{1,2} b_{2,1} b_{3,3} .
\end{aligned}
$$

Expanding $b_{1,1} b_{2,2} b_{3,3}$ and omitting terms with repeated indeterminates, we have

$$
\begin{aligned}
{\left[z_{G}\right] b_{1,1} b_{2,2} b_{3,3}=} & {\left[z_{G}\right]\left(z_{1,1,1} z_{1,1,2} z_{1,3,1} z_{1,3,2} z_{2,1,1} z_{2,1,2} z_{2,2,1} z_{2,2,2} z_{2,3,1} z_{2,3,2} z_{3,2,1} z_{3,2,2}\right.} \\
& \left.+z_{1,1,1} z_{2,1,2} z_{2,2,1} z_{2,2,2} z_{2,3,1} z_{1,3,2} z_{2,1,1} z_{1,1,2} z_{1,3,1} z_{2,3,2} z_{3,2,1} z_{3,2,2}\right)
\end{aligned}
$$

Sorting indeterminates into lexicographic order and using (3.14), we see that this is

$$
\left[z_{G}\right]\left(z_{G}+q z_{G}\right)=1+q .
$$

Similarly computing $\left[z_{G}\right] b_{1,2} b_{2,1} b_{3,3}$, we obtain $\left(q^{\frac{1}{2}}+q^{\frac{3}{2}}\right)$. Thus Eq. (3.16) gives

$$
\sigma_{B}\left(x_{2,2} x_{1,1} x_{3,3}\right)=(1+q)+\left(q^{\frac{1}{2}}-q^{-\frac{1}{2}}\right)\left(q^{\frac{1}{2}}+q^{\frac{3}{2}}\right)=q+q^{2}
$$

For special combinations of $u, v \in \mathfrak{S}_{n}$ and an expression $s_{i_{1}} \cdots s_{i_{m}}$, there are simple rules for computing $\sigma_{B}\left(x^{u, v}\right)$.

Proposition 3.2 Fix $u, v \in \mathfrak{S}_{n}$, and an expression $s_{i_{1}} \cdots s_{i_{\ell}}$ whose wiring diagram has weighted path matrix $B$. Then we have the following.

(i) $\sigma_{B}\left(x^{u, v}\right)=0$ unless $s_{i_{1}} \cdots s_{i_{\ell}}$ contains a subexpression for $u^{-1} v$.

(ii) If $s_{i_{1}} \cdots s_{i_{\ell}}$ is a reduced expression for $w \in \mathfrak{S}_{n}$, then $\sigma_{B}\left(x^{e, w}\right)=q w$, and $\sigma_{B}\left(x^{e, v}\right)=0$ unless $v \leq w$.

Proof (i) Suppose that $b_{u_{1}, v_{1}} \cdots b_{u_{n}, v_{n}} \neq 0$. Then there is a path family $\pi$ of type $u^{-1} v$ which covers the wiring diagram $G$ of $s_{i_{1}} \cdots s_{i_{\ell}}$. Define the binary word $\beta=\beta_{1} \cdots \beta_{\ell}$ by

$$
\beta_{j}= \begin{cases}1 & \text { if two paths of } \pi \text { cross at the central vertex of } G_{\left[i_{j}, i_{j}+1\right]} \\ 0 & \text { otherwise. }\end{cases}
$$

Then we have type $(\pi)=s_{i_{1}}^{\beta_{1}} \cdots s_{i_{\ell}}^{\beta_{\ell}}$ and the factors with $\beta_{j}=1$ form a subexpression of $s_{i_{1}} \cdots s_{i_{\ell}}$ which is equal in $\mathfrak{S}_{n}$ to type $(\pi)$.

(ii) If $s_{i_{1}} \cdots s_{i_{\ell}}$ is reduced, then there is exactly one path family $\pi$ which covers $G$ and has type $u^{-1} v$. Its component paths cross at the central vertex of each simple transposition graph $G_{\left[i_{j}, i_{j}+1\right]}$. Each crossing causes the variables $z_{i_{j}, j, 1}, z_{i_{j}+1, j, 2}$ to appear earlier than the variables $z_{i_{j}+1, j, 1}, z_{i_{j}, j, 2}$, contributing $q^{\frac{1}{2}}$ to $\sigma_{B}\left(x^{e, w}\right)$. Now 
consider a permutation $v$ with $v \not \leq w$. By definition of the Bruhat order (1.2) there is no subexpression of $s_{i_{1}} \cdots s_{i_{\ell}}$ which is an expression for $v$. By (i.) we have $\sigma_{B}\left(x^{e, v}\right)=0$.

An important special case of Proposition 3.2 concerns wiring diagrams for a single generator.

Corollary 3.3 Let wiring diagram $H$ of the reduced expression $s_{i_{1}}=s_{j}$ have weighted path matrix $C$. For $u, v \in \mathfrak{S}_{n}$ we have

$$
\sigma_{C}\left(x^{u, v}\right)= \begin{cases}q^{\frac{1}{2}} & \text { if } u=v s_{j}, \\ q & \text { if } u=v \text { and } v s_{j}<v, \\ 1 & \text { if } u=v \text { and } v s_{j}>v, \\ 0 & \text { if } u \notin\left\{v, v s_{j}\right\} .\end{cases}
$$

Proof The matrix $C$ is obtained from the $n \times n$ identity matrix by replacing its $[j, j+1]$, $[j, j+1]$ submatrix by

$$
\left[\begin{array}{cc}
z_{j, 1,1} z_{j, 1,2} & z_{j, 1,1} z_{j+1,1,2} \\
z_{j+1,1,1} z_{j, 1,2} & z_{j+1,1,1} z_{j+1,1,2}
\end{array}\right]
$$

and we have $z_{H}=z_{j, 1,1} z_{j, 1,2} z_{j+1,1,1} z_{j+1,1,2}$. Using Proposition 2.1 to expand $x^{u, v}$ in the natural basis of $\mathcal{A}_{[n],[n]}$ and recalling that Proposition 3.2 (ii) implies $\sigma_{C}\left(x^{e, w}\right)$ to vanish unless $w \in\left\{e, s_{j}\right\}$, we can write

$$
\sigma_{C}\left(x^{u, v}\right)=\sigma_{C}\left(x^{e, u^{-1} v}\right)+\sum_{\substack{w>u^{-1} v \\ w \leq s_{j}}} r_{u, v, e, w}\left(q^{\frac{1}{2}}-q^{-\frac{1}{2}}\right) \sigma_{C}\left(x^{e, w}\right) .
$$

Now observe that the restrictions on $w$ imply this expression to vanish unless $u \in$ $\left\{v, v s_{j}\right\}$. When $u=v s_{j}$ we have

$$
\begin{aligned}
\sigma_{C}\left(x^{v s_{j}, v}\right) & =\sigma_{C}\left(x^{e, s_{j}}\right)=\left[z_{H}\right] z_{j, 1,1} z_{j+1,1,2} z_{j+1,1,1} z_{j, 1,2} \\
& =\left[z_{H}\right] q^{\frac{1}{2}} z_{j, 1,1} z_{j, 1,2} z_{j+1,1,2} z_{j+1,1,1}=q^{\frac{1}{2}},
\end{aligned}
$$

since $z_{j, 1,2}$ commutes with $z_{j+1,1,1}$ and quasicommutes with $z_{j+1,1,2}$. On the other hand, when $u=v$, we may use (2.13) and (3.17) to obtain

$$
\begin{aligned}
\sigma_{C}\left(x^{v, v}\right) & =\sigma_{C}\left(x^{e, e}\right)+r_{v, v, e, s_{j}}\left(q^{\frac{1}{2}}-q^{-\frac{1}{2}}\right) \sigma_{C}\left(x^{e, s_{j}}\right) \\
& =\left[z_{H}\right] z_{j, 1,1} z_{j, 1,2} z_{j+1,1,1} z_{j+1,1,2}+q^{\frac{1}{2}} \begin{cases}q^{\frac{1}{2}}-q^{-\frac{1}{2}} & \text { if } v s_{j}<v, \\
0 & \text { otherwise }\end{cases} \\
& = \begin{cases}q & \text { if } v s_{j}<v, \\
1 & \text { otherwise. }\end{cases}
\end{aligned}
$$


The map $\sigma_{B}$ behaves well with respect to concatenation of wiring diagrams.

Proposition 3.4 Let wiring diagrams $G$, $H$ of expressions $s_{i_{1}} \cdots s_{i_{k}}, s_{i_{k+1}} \cdots s_{i_{m}}$ have weighted path matrices $B, C$, respectively. Then for all $u, w \in \mathfrak{S}_{n}$ we have

$$
\sigma_{B C}\left(x^{u, w}\right)=\sum_{v \in \mathfrak{S}_{n}} \sigma_{B}\left(x^{u, v}\right) \sigma_{C}\left(x^{v, w}\right) .
$$

Proof Write $A=\left(a_{i, j}\right)=B C$ for the weighted path matrix of $G \circ H$, and consider

$$
\begin{aligned}
\sigma_{B C}\left(x^{u, w}\right) & =\left[z_{G \circ H}\right] a_{u_{1}, w_{1}} \cdots a_{u_{n}, w_{n}} \\
& =\left[z_{G \circ H}\right]\left(\sum_{j_{1}=1}^{n} b_{u_{1}, j_{1}} c_{j_{1}, w_{1}}\right) \cdots\left(\sum_{j_{n}=1}^{n} b_{u_{n}, j_{n}} c_{j_{n}, w_{n}}\right) .
\end{aligned}
$$

In all but $n$ ! of the $n^{n}$ resulting terms, repeated indices among $j_{1}, \ldots, j_{n}$ lead to repeated indeterminates or matrix entries equal to 0 , which cause the coefficient of $z_{G \circ H}$ to be 0 . Thus we may consider only the $n$ ! terms in which $j_{1}, \ldots, j_{n}$ are all distinct, and the expression reduces to

$$
\left[z_{G \circ H}\right] \sum_{v \in \mathfrak{S}_{n}} b_{u_{1}, v_{1}} c_{v_{1}, w_{1}} \cdots b_{u_{n}, v_{n}} c_{v_{n}, w_{n}} .
$$

Now observe that $b_{u_{i}, v_{i}}$ commutes with $c_{v_{j}, w_{j}}$ for all $i, j$, since our indexing of the expressions $s_{i_{1}} \cdots s_{i_{k}}, \quad s_{i_{k+1}} \cdots s_{i_{m}}$ guarantees all edge weights $\left\{z_{i_{j}, j, 1}, z_{i_{j}, j, 2}, z_{i_{j}+1, j, 1}, z_{i_{j}+1, j, 2} \mid 1 \leq j \leq k\right\}$ of $G$ to commute with all edge weights $\left\{z_{i_{j}, j, 1}, z_{i_{j}, j, 2}, z_{i_{j}+1, j, 1}, z_{i_{j}+1, j, 2} \mid k+1 \leq j \leq m\right\}$ of $H$. Thus (3.20) is equal to

$$
\begin{aligned}
\sum_{v \in \mathfrak{S}_{n}}\left[z_{G \circ H}\right] b_{u_{1}, v_{1}} \cdots b_{u_{n}, v_{n}} c_{v_{1}, w_{1}} \cdots c_{v_{n}, w_{n}} & =\sum_{v \in \mathfrak{S}_{n}}\left[z_{G}\right] b_{u_{1}, v_{1}} \cdots b_{u_{n}, v_{n}}\left[z_{H}\right] c_{v_{1}, w_{1}} \cdots c_{v_{n}, w_{n}} \\
& =\sum_{v \in \mathfrak{S}_{n}} \sigma_{B}\left(x^{u, v}\right) \sigma_{C}\left(x^{v, w}\right) .
\end{aligned}
$$

The special case of Proposition 3.4 in which we have $m=k+1$ (so that $H$ is the wiring diagram of the single generator $\left.s_{i_{k+1}}\right)$ leads to a simple formula for $\sigma_{B C}\left(x^{u, w}\right)$ in terms of the matrix $B$.

Corollary 3.5 Let wiring diagrams $G$, $H$ of expressions $s_{i_{1}} \cdots s_{i_{k}}$, $s_{i_{k+1}}$ have weighted path matrices $B, C$, respectively. Then for all $w \in \mathfrak{S}_{n}$ we have

$$
\sigma_{B C}\left(x^{u, w}\right)=q^{\frac{1}{2}} \sigma_{B}\left(x^{u, w s_{i}} i_{k+1}\right)+ \begin{cases}q \sigma_{B}\left(x^{u, w}\right) & \text { if } w s_{i_{k+1}}<w \\ \sigma_{B}\left(x^{u, w}\right) & \text { if } w s_{i_{k+1}}>w\end{cases}
$$


Proof By Corollary 3.3, the expansion in Proposition 3.4 has only two nonzero terms:

$$
\sigma_{B C}\left(x^{u, w}\right)=\sigma_{B}\left(x^{u, w s_{i_{k+1}}}\right) \sigma_{C}\left(x^{w s_{i_{k+1}}, w}\right)+\sigma_{B}\left(x^{u, w}\right) \sigma_{C}\left(x^{w, w}\right) .
$$

Evaluating $\sigma_{C}$ as in Corollary 3.3 we then have the desired expression.

Another important property of the map $\sigma_{B}$ is that its evaluation at natural basis elements of $\mathcal{A}_{[n],[n]}$ is closely related to coefficients in the natural expansion of (1+ $\left.T_{s_{i_{1}}}\right) \cdots\left(1+T_{s_{i_{m}}}\right)$ in $H_{n}(q)$.

Proposition 3.6 Let $G$ be the wiring diagram in (3.3) with weighted path matrix $B$, and fix $w \in \mathfrak{S}_{n}$. Then $\sigma_{B}\left(x^{e, w}\right)$ is equal to $q_{w}$ times the coefficient of $T_{w}$ in $(1+$ $\left.T_{s_{i_{1}}}\right) \cdots\left(1+T_{s_{i_{m}}}\right)$.

Proof Consider the wiring diagram $G=G_{[j, j+1]}$ of the simple transposition $s_{j}$ and its weighted path matrix $B$. By Corollary 3.3 we have

$$
\sigma_{B}\left(x^{e, w}\right)= \begin{cases}q^{\frac{1}{2}} & \text { if } w=s_{j} \\ 1 & \text { if } w=e \\ 0 & \text { otherwise }\end{cases}
$$

Thus the result is true for any simple transposition wiring diagram in (3.2).

Now assume that the result holds for concatenations of $1, \ldots, m-1$ simple transposition diagrams, and define $\left\{a_{w} \mid w \in \mathfrak{S}_{n}\right\} \subset \mathbb{Z}[q]$ by

$$
\left(1+T_{s_{i_{1}}}\right) \cdots\left(1+T_{s_{i_{m-1}}}\right)=\sum_{w \in \mathfrak{S}_{n}} a_{w} T_{w}
$$

Consider the wiring diagram $G=G_{\left[i_{1}, i_{1}+1\right]} \circ \cdots \circ G_{\left[i_{m}, i_{m}+1\right]}$ and decompose $G$ as $G^{\prime} \circ H$, where $G^{\prime}=G_{\left[i_{1}, i_{1}+1\right]} \circ \cdots \circ G_{\left[i_{m-1}, i_{m-1}+1\right]}$ has weighted path matrix $B^{\prime}$ and $H=G_{\left[i_{m}, i_{m}+1\right]}$ has weighted path matrix $C$. By Corollary 3.5 we have

$$
\sigma_{B}\left(x^{e, w}\right)=\sigma_{B^{\prime} C}\left(x^{e, w}\right)=q^{\frac{1}{2}} \sigma_{B^{\prime}}\left(x^{e, w s_{i_{m}}}\right)+ \begin{cases}q \sigma_{B^{\prime}}\left(x^{e, w}\right) & \text { if } w s_{i_{m}}<w \\ \sigma_{B^{\prime}}\left(x^{e, w}\right) & \text { if } w s_{i_{m}}>w .\end{cases}
$$

By induction, this is

$q^{\frac{1}{2}} q_{w s_{i_{m}}} a_{w s_{i_{m}}}+\left\{\begin{array}{ll}q q_{w} a_{w} & \text { if } w s_{i_{m}}<w \\ q_{w} a_{w} & \text { if } w s_{i_{m}}>w\end{array}= \begin{cases}q_{w}\left(a_{w s_{i_{m}}}+q a_{w}\right) & \text { if } w s_{i_{m}}<w \\ q_{w}\left(q a_{w s_{i_{m}}}+a_{w}\right) & \text { if } w s_{i_{m}}>w\end{cases}\right.$

On the other hand, consider the element

$$
\left(1+T_{s_{i_{1}}}\right) \cdots\left(1+T_{s_{i_{m}}}\right)=\left(\sum_{v \in \mathfrak{S}_{n}} a_{v} T_{v}\right)\left(1+T_{s_{i_{m}}}\right)
$$


By (1.1) we have

$$
T_{w} T_{s_{i_{m}}}= \begin{cases}(q-1) T_{w}+q T_{w s_{i_{m}}} & \text { if } w s_{i_{m}}<w \\ T_{w s_{i_{m}}} & \text { if } w s_{i_{m}}>w\end{cases}
$$

Thus $q_{w}$ times the coefficient of $T_{w}$ in (3.21) is

$$
q_{w}\left(a_{w}+\left\{\begin{array}{ll}
a_{w s_{i m}}+(q-1) a_{w} & \text { if } w s_{i_{m}}<w \\
q a_{w s_{i_{m}}} & \text { if } w s_{i_{m}}>w
\end{array}\right)= \begin{cases}q_{w}\left(a_{w s_{i_{m}}}+q a_{w}\right) & \text { if } w s_{i_{m}}<w \\
q_{w}\left(q a_{w s_{i_{m}}}+a_{w}\right) & \text { if } w s_{i_{m}}>w\end{cases}\right.
$$

As a consequence of Proposition 3.6, we have a $q$-analog of Proposition 3.1. The evaluation

$$
B \mapsto \operatorname{Imm}_{\theta}(B)
$$

of $\operatorname{Imm}_{\theta}(x) \in \mathbb{Z}\left[x_{1,1}, \ldots, x_{n, n}\right]$ at the $n \times n$ matrix $B$ is now replaced by the map

$$
B \mapsto \sigma_{B}\left(\operatorname{Imm}_{\theta_{q}}(x)\right)
$$

for $\operatorname{Imm}_{\theta_{q}}(x) \in \mathcal{A}$.

Theorem 3.7 Let $\theta_{q}: H_{n}(q) \rightarrow \mathbb{Z}\left[q^{\frac{1}{2}}, q^{-\frac{1}{2}}\right]$ be linear, and let wiring diagram $G$ of $s_{i_{1}} \cdots s_{i_{m}}$ have weighted path matrix $B$. Then we have

$$
\theta_{q}\left(\left(1+T_{s_{i_{1}}}\right) \cdots\left(1+T_{s_{i_{m}}}\right)\right)=\sigma_{B}\left(\operatorname{Imm}_{\theta_{q}}(x)\right)
$$

Proof Write $\left(1+T_{s_{i_{1}}}\right) \cdots\left(1+T_{s_{i_{m}}}\right)=\sum_{v \in \mathfrak{S}_{n}} a_{v} T_{v}$. Then the right-hand side of (3.22) is

$$
\begin{aligned}
\sigma_{B}\left(\sum_{v \in \mathfrak{S}_{n}} \theta_{q}\left(T_{v}\right) q_{v}^{-1} x^{e, v}\right) & =\sum_{v \in \mathfrak{S}_{n}} \theta_{q}\left(T_{v}\right) q_{v}^{-1} \sigma_{B}\left(x^{e, v}\right) \\
& =\sum_{v \in \mathfrak{S}_{n}} \theta_{q}\left(T_{v}\right) q_{v}^{-1} q_{v} a_{v} \\
& =\theta_{q}\left(\sum_{v \in \mathfrak{S}_{n}} a_{v} T_{v}\right)
\end{aligned}
$$

where the second equality follows from Proposition 3.6. But this is precisely the lefthand side of (3.22). 
Now observe that if one fixes a reduced expression $s_{i_{1}} \cdots s_{i_{m}}$ for each $w \in \mathfrak{S}_{n}$ and uses each such expression to define an element

$$
D_{w} \underset{\mathrm{def}}{=}\left(1+T_{s_{i_{1}}}\right) \cdots\left(1+T_{s_{i_{m}}}\right) \in H_{n}(q)
$$

then the set $\left\{D_{w} \mid w \in \mathfrak{S}_{n}\right\}$ forms a basis of $H_{n}(q)$ : we have $D_{w} \in T_{w}+$ $\operatorname{span}_{\mathbb{Z}[q]}\left\{T_{v} \mid v<w\right\}$ (see also [5, Cor.3.6]). Thus we can evaluate $\theta_{q}(g)$ for every $g \in H_{n}(q)$, provided that we can expand $g$ in this basis.

\section{G-tableaux and the combinatorics of the evaluation map}

Theorem 3.7 provides half of the solution to the problem of evaluating $\epsilon_{q}^{\lambda}\left(D_{w}\right)$. The other half is a combinatorial interpretation of the right-hand-side of (3.22), which is a linear combination of expressions of the form $\sigma_{B}\left(x^{u, w}\right) \in \mathbb{Z}\left[q^{\frac{1}{2}}, q^{-\frac{1}{2}}\right]$. To combinatorially interpret such evaluations, we will arrange the paths of a path family $\pi$ covering a wiring diagram $G$ into a (French) Young diagram. We will call the resulting structure a $G$-tableau, or more specifically a $\pi$-tableau. If type $(\pi)=w$, we will say also that the tableau has type $w$. For example, the following path family $\pi$ covering the wiring diagram of (3.3) yields six $\pi$-tableaux of shape 21 and type 213 :

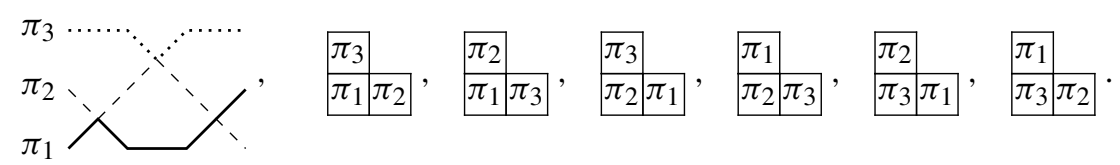

Given a $\pi$-tableau $U$, we define (integer) Young tableaux $L(U), R(U)$ by replacing each path by its source index and sink index, respectively. For example, if $U$ is the first $\pi$-tableau in (4.1), then we have

$$
L(U)=\begin{array}{|l|l|l}
\hline 3 &
\end{array}, \quad R(U)=\begin{array}{|l|l|}
\hline 3 & \\
\hline 1 & 2 \\
\hline 2 & 1 \\
\hline
\end{array} .
$$

It is easy to see that given two Young tableaux $P, Q$ of the same shape, there is at most one $\pi$-tableau $U$ satisfying $L(U)=P, R(U)=Q$.

We will also define several statistics on $G$-tableaux. Let $U$ be a $\pi$-tableau of any shape $\lambda \vdash n$. Define $\operatorname{INVNC}(U)$, the number of inverted noncrossings of $U$, to be the number of noncrossings $j$ of $\pi$ such that $\pi_{a}, \pi_{b}$ intersect at the central vertex of $G_{\left[i_{j}, i_{j}+1\right]}$ (3.3) with $\pi_{b}$ above $\pi_{a}$,

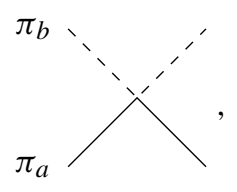


and $\pi_{b}$ appears in an earlier column of $U$ than $\pi_{a}$ (whether or not $b>a$ ). Thus inverted noncrossings may be proper or defective. Define $\mathrm{C}(U)=\mathrm{C}(\pi)$ to be the number of crossings of $\pi$, i.e., the number of occurrences of

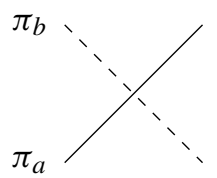

or

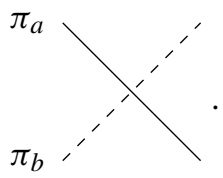

This depends only upon $\pi$; not upon the locations of $\pi_{a}$ and $\pi_{b}$ in $U$. For example, each tableau $U$ in (4.1) satisfies $\mathrm{C}(U)=1$ because $\mathrm{C}(\pi)=1$. The inverted noncrossings in these tableaux are appearances of $\pi_{3}$ in an earlier column than $\pi_{2}$, or $\pi_{2}$ in an earlier column than $\pi_{1}$. The numbers of these for the six tableaux are $1,0,0,0,1,1$, respectively.

Combining the above tableau statistics, we have a combinatorial interpretation of $\sigma_{B}\left(x^{u, w}\right)$.

Proposition 4.1 Let wiring diagram $G$ have weighted path matrix $B$. For $u, w \in \mathfrak{S}_{n}$ we have

$$
\sigma_{B}\left(x^{u, w}\right)=\sum_{\pi} q^{\frac{\mathrm{C}(\pi)}{2}} q^{\mathrm{INVNC}(U)}
$$

where the sum is over path families $\pi$ of type $u^{-1} w$ covering $G$, and $U=U(\pi, u, w)$ is the unique $\pi$-tableaux of shape $(n)$ satisfying $L(U)=u_{1} \cdots u_{n}, R(U)=w_{1} \cdots w_{n}$.

Proof Let $G=G_{\left[i_{1}, i_{1}+1\right]} \circ \cdots \circ G_{\left[i_{m}, i_{m}+1\right]}$. If $s_{i_{1}} \cdots s_{i_{m}}$ contains no reduced subexpression for $u^{-1} w$, then there is no path family of type $u^{-1} w$ which covers $G$, and the right-hand side of (4.4) is 0. By Proposition 3.2, the left-hand side is 0 as well. Now suppose $m=\ell\left(u^{-1} w\right)$ and let $s_{i_{1}} \cdots s_{i_{m}}$ be a reduced expression for $u^{-1} w$. Then there is exactly one path family of type $u^{-1} w$ that covers $G$. It has $\ell\left(u^{-1} w\right)$ crossings and no noncrossings. Thus the right-hand side of (4.4) is $q_{u^{-1} w}$. By Proposition 2.1 and Proposition 3.2, the left-hand side is the same. Thus the claim is true for wiring diagrams $G$ which are concatenations of $0, \ldots, \ell\left(u^{-1} w\right)$ simple transposition diagrams.

Now suppose the claim is true for $G$ a concatenation of $m \geq \ell\left(u^{-1} w\right)$ simple transposition diagrams and consider $G=G^{\prime} \circ H$ where

$$
G^{\prime}=G_{\left[i_{1}, i_{1}+1\right]} \circ \cdots \circ G_{\left[i_{m}, i_{m}+1\right]}, \quad H=G_{\left[i_{m+1}, i_{m+1}+1\right]} .
$$

Let $B^{\prime}, C$ be the path matrices of $G^{\prime}, H$, respectively so that $B=B^{\prime} C$ is the path matrix of $G$. Then by Corollary 3.5 we have

$$
\sigma_{B}\left(x^{u, v}\right)=q^{\frac{1}{2}} \sigma_{B^{\prime}}\left(x^{u, w s_{i_{m+1}}}\right)+ \begin{cases}q \sigma_{B^{\prime}}\left(x^{u, w}\right) & \text { if } w s_{i_{m+1}}<w \\ \sigma_{B^{\prime}}\left(x^{u, w}\right) & \text { if } w s_{i_{m+1}}>w\end{cases}
$$


By induction we may interpret $\sigma_{B^{\prime}}\left(x^{u, w s_{i m+1}}\right)$ and $\sigma_{B^{\prime}}\left(x^{u, w}\right)$, respectively, as

$$
\sum_{\pi^{B^{\prime}}} q^{\frac{\mathrm{C}\left(\pi^{B^{\prime}}\right)}{2}} q^{\operatorname{INVNC}\left(U\left(\pi^{B^{\prime}}, u, w s_{i m+1}\right)\right)}, \quad \sum_{\pi^{B^{\prime}}} q^{\frac{\mathrm{C}\left(\pi^{B^{\prime}}\right)}{2}} q^{\operatorname{INVNC}\left(U\left(\pi^{B^{\prime}}, u, w\right)\right)}
$$

where the sums are over path families of type $u^{-1} w s_{i_{m+1}}$ and $u^{-1} w$, respectively, which cover the wiring diagram $G^{\prime}$. Substituting these two expressions into the right-hand side of (4.5), we obtain

$$
\sum_{\pi^{B^{\prime}}} q^{\frac{\mathrm{C}\left(\pi^{B^{\prime}}\right)+1}{2}} q^{\operatorname{INVNC}\left(U\left(\pi^{B^{\prime}}, u, w s_{i_{m+1}}\right)\right)}+ \begin{cases}\sum_{\pi^{B^{\prime}}} q^{\frac{\mathrm{C}\left(\pi^{B^{\prime}}\right)+2}{2}} q^{\operatorname{INVNC}\left(U\left(\pi^{B^{\prime}}, u, w\right)\right)} & \text { if } w s_{i_{m+1}}<w, \\ \sum_{\pi^{B^{\prime}}} q^{\frac{\mathrm{C}\left(\pi^{B^{\prime}}\right)}{2}} q^{\operatorname{INVNC}\left(U\left(\pi^{B^{\prime}}, u, w\right)\right)} & \text { if } w s_{i_{m+1}}>w,\end{cases}
$$

where the sums are as in (4.6).

Concatenating a path family $\pi^{B^{\prime}}$ which covers $G^{\prime}$ to a path family $\pi^{C}$ which covers $H$ we obtain a new path family $\pi^{B^{\prime} C}$ which covers $G$ and satisfies type $\left(\pi^{B^{\prime} C}\right)=$ type $\left(\pi^{B^{\prime}}\right)$ type $\left(\pi^{C}\right)$. Conversely, every path family which covers $G$ decomposes this way. If type $\left(\pi^{B^{\prime}}\right)=u^{-1} w s_{i_{m+1}}$ and type $\left(\pi^{C}\right)=s_{i_{m+1}}$, then we have

$$
\operatorname{INVNC}\left(U\left(\pi^{B^{\prime} C}, u, w\right)\right)=\operatorname{INVNC}\left(U\left(\pi^{B^{\prime}}, u, w s_{i_{m+1}}\right)\right), \quad \mathrm{C}\left(\pi^{B^{\prime} C}\right)=\mathrm{C}\left(\pi^{B^{\prime}}\right)+1 .
$$

Otherwise, if type $\left(\pi^{B^{\prime}}\right)=u^{-1} w$ and type $\left(\pi^{C}\right)=e$, then let $j$ and $k$ be the source indices of the paths in $\pi^{B^{\prime}}$ which terminate at sinks $i_{m+1}$ and $i_{m+1}+1$, respectively. Then we have

$$
\begin{aligned}
\operatorname{INVNC}\left(U\left(\pi^{B^{\prime} C}, u, w\right)\right) & =\operatorname{INVNC}\left(U\left(\pi^{B^{\prime}}, u, w\right)\right)+ \begin{cases}1 & \text { if } k \text { precedes } j \text { in } u, \text { i.e., } u_{k}^{-1}<u_{j}^{-1}, \\
0 & \text { otherwise, }\end{cases} \\
\mathrm{C}\left(\pi^{B^{\prime} C}\right) & =\mathrm{C}\left(\pi^{B^{\prime}}\right) .
\end{aligned}
$$

Since type $\left(\pi^{B^{\prime}}\right)=u^{-1} w$, the index $k$ is given by $k=\left(u^{-1} w\right)_{i_{m+1}+1}^{-1}=\left(w^{-1} u\right)_{i_{m+1}+1}$. Thus the first condition in (4.9) is equivalent to

$$
\left(u^{-1}\right)_{\left(w^{-1} u\right)_{i_{m+1}+1}}<\left(u^{-1}\right)_{\left(w^{-1} u\right)_{i_{m+1}}} .
$$

Simplifying the two expressions in this inequality to $\left(w^{-1} u u^{-1}\right)_{i_{m+1}+1}=\left(w^{-1}\right)_{i_{m+1}+1}$ and $\left(w^{-1} u u^{-1}\right)_{i_{m+1}}=\left(w^{-1}\right)_{i_{m+1}}$, respectively, we obtain the equivalent inequality $w s_{i_{m+1}}<w$.

It follows that the expression in (4.7) and therefore the right-hand side of (4.5) can be written as

$$
\sum_{\pi^{B^{\prime} C}} q^{\frac{\mathrm{C}\left(\pi^{B^{\prime} C}\right)}{2}} q^{\mathrm{INVNC}\left(U\left(\pi^{B^{\prime} C}, u, w\right)\right)},
$$


where the sum is over path families of type $u^{-1} w$ which cover $G$. Thus the claim is true by induction.

The special case $u=e$ of Proposition 4.1 yields another proof of the formula (3.6).

Corollary 4.2 The coefficients in the expansion $\left(1+T_{s_{i_{1}}}\right) \cdots\left(1+T_{s_{i_{m}}}\right)=\sum_{w} a_{w} T_{w}$ are given by

$$
a_{w}=\sum_{\pi} q^{\mathrm{D}(\pi)}
$$

where the sum is over all path families of type $w$ which cover the wiring diagram of $s_{i_{1}} \cdots s_{i_{m}}$.

Proof Substitute $u=e$ in Proposition 4.1.

The right-hand side of (4.4) is a sum over path families $\pi$ of type $w$, and each tableau $U=U(\pi, e, w)$ is simply the sequence $\pi=\left(\pi_{1}, \ldots, \pi_{n}\right)$. Thus an inverted noncrossing of $U$ is simply a noncrossing (4.2) in which the upper path has index less than that of the lower path, i.e., a defective noncrossing. To relate these to all defects, let us temporarily define

$$
\begin{aligned}
\operatorname{DNC}(\pi) & =\text { number of defective noncrossings of } \pi, \\
\operatorname{DC}(\pi) & =\text { number of defective crossings of } \pi,
\end{aligned}
$$

so that $\mathrm{D}(\pi)=\operatorname{DNC}(\pi)+\mathrm{DC}(\pi)$. Now observe that for any path family of type $w$ we have

$$
\mathrm{C}(\pi)=\operatorname{INV}(w)+2 \mathrm{DC}(\pi)
$$

because if paths $\pi_{a}, \pi_{b}$ cross $k$ times, then at most one of those crossings contributes to $\operatorname{INV}(w)$, while exactly half of the remaining crossings are defective. Thus the righthand side of (4.4) becomes

$$
\sum_{\pi} q^{\frac{\mathrm{INV}(w)+2 \mathrm{DC}(\pi)}{2}} q^{\mathrm{DNC}(\pi)}=\sum_{\pi} q_{w} q^{\mathrm{DC}(\pi)+\mathrm{DNC}(\pi)}=q_{w} \sum_{\pi} q^{\mathrm{D}(\pi)},
$$

where the sum is over all path families of type $w$ which cover the wiring diagram of $s_{i_{1}} \cdots s_{i_{m}}$.

By Proposition 3.6, the left-hand side of (4.4) is $q_{w} a_{w}$. Combining this with (4.11), we have the desired result.

\section{Evaluation of induced sign characters}

By Theorem 3.7, the map $\sigma_{B}$ (3.15) can be used to evaluate $\epsilon_{q}^{\lambda}\left(D_{w}\right)$ when one has a simple expression for the generating function $\operatorname{Imm}_{\epsilon_{q}^{\lambda}}(x)$ and can evaluate $\sigma_{B}\left(\operatorname{Imm}_{\epsilon_{q}^{\lambda}}(x)\right)$. 
Such an expression was given by Konvalinka and the third author in [9, Thm. 5.4]: for $\lambda=\left(\lambda_{1}, \ldots, \lambda_{r}\right)$, we have

$$
\operatorname{Imm}_{\epsilon_{q}^{\lambda}}(x)=\sum_{I} \operatorname{det}_{q}\left(x_{I_{1}, I_{1}}\right) \cdots \operatorname{det}_{q}\left(x_{I_{r}, I_{r}}\right)
$$

where $\operatorname{det}_{q}$ and $x_{L, M}$ are defined as in Sect. 2, and the sum is over all ordered set partitions $I=\left(I_{1}, \ldots, I_{r}\right)$ of $[n]$ satisfying $\left|I_{j}\right|=\lambda_{j}$. We will say that such an ordered set partition has type $\lambda$.

To evaluate $\sigma_{B}\left(\operatorname{Imm}_{\epsilon_{q}^{\lambda}}(x)\right)$, we expand each term on the right-hand side of (5.1) in a monomial basis $\left\{x^{u, v} \mid v \in \mathfrak{S}_{n}\right\}$ of $\mathcal{A}_{[n],[n]}$, where $u=u(I)$ is the concatenation of the $r$ strictly increasing subwords

$$
u_{1} \cdots u_{\lambda_{1}}, \quad u_{\lambda_{1}+1} \cdots u_{\lambda_{1}+\lambda_{2}}, \quad u_{\lambda_{1}+\lambda_{2}+1} \cdots u_{\lambda_{1}+\lambda_{2}+\lambda_{3}}, \quad \cdots, \quad u_{n-\lambda_{r}+1} \cdots u_{n}
$$

formed by listing the elements of each block $I_{1}, \ldots, I_{r}$ in increasing order. As $I$ varies over all ordered set partitions of $[n]$ of type $\lambda$, the permutations $u(I)$ vary over the Bruhat-minimal representatives $\mathfrak{S}_{\lambda}^{-}$of cosets $\mathfrak{S}_{\lambda} u$, where $\mathfrak{S}_{\lambda}$ is the Young subgroup of $\mathfrak{S}_{n}$ generated by

$$
\left\{s_{1}, \ldots, s_{n-1}\right\} \backslash\left\{s_{\lambda_{1}}, s_{\lambda_{1}+\lambda_{2}}, s_{\lambda_{1}+\lambda_{2}+\lambda_{3}}, \ldots, s_{n-\lambda_{r}}\right\} .
$$

Expanding each term on the right-hand side of (5.1) and applying $\sigma_{B}$ we have

$$
\sigma_{B}\left(\operatorname{det}_{q}\left(x_{I_{1}, I_{1}}\right) \cdots \operatorname{det}_{q}\left(x_{I_{r}, I_{r}}\right)\right)=\sum_{y \in \mathfrak{S}_{\lambda}}(-1)^{\ell(y)} q_{y}^{-1} \sigma_{B}\left(x^{u(I), y u(I)}\right) .
$$

To combinatorially interpret the sum in (5.3) we may apply Proposition 4.1 and compute statistics for tableaux belonging to the set

$$
\mathcal{U}_{I}=\mathcal{U}_{I}(G) \underset{\text { def }}{=}\left\{U(\pi, u, y u) \mid \pi \text { covers } G, u=u(I), \text { type }(\pi)=y \in \mathfrak{S}_{\lambda}\right\}
$$

Note that our restriction on $y$ forces the sink indices of paths in components

$$
\left(\lambda_{1}+\cdots+\lambda_{k-1}+1\right), \ldots,\left(\lambda_{1}+\cdots+\lambda_{k}\right)
$$

of $U(\pi, u, y u)$ to be a permutation of the source indices of the same paths.

On the other hand, the sum in (5.3) has both positive and negative signs. We will obtain a subtraction-free expression for the sum by applying a sign-reversing involution to the tableaux in each set $\mathcal{U}_{I}$. It will be convenient to define this involution on a second set $\mathcal{T}_{I}$ of tableaux, in obvious bijection $\mathcal{U}_{I}$. For a wiring diagram $G$ let $\mathcal{T}_{I}=\mathcal{T}_{I}(G)$ be the set of all column-closed, left column-strict $G$-tableaux $W$ of shape $\lambda^{\top}$ such that $L\left(W^{\top}\right)_{k}=I_{k}$ (as sets) for $k=1, \ldots, r$. The bijection $\delta=\delta_{I}: \mathcal{U}_{I} \rightarrow \mathcal{T}_{I}$ maps $U \in \mathcal{U}$ to the left column-strict $G$-tableau $W$ of shape $\lambda^{\top}$ whose $k$ th column consists of entries (5.4) of $U$. 
Since $U$ and $\delta(U)$ contain the same path family, it is easy to see that $\delta$ does not affect the statistic C. On the other hand, it changes the statistic INVNC in a very simple way. Define $\operatorname{CDNC}(U)$ to be the number of defective noncrossings of pairs of paths appearing in the same column of $U$, i.e., the number of occurrences of (4.2) where $b<a$ and $\pi_{b}, \pi_{a}$ appear in the same column of $U$.

Lemma 5.1 Let I be an ordered set partition. For $U \in \mathcal{U}_{I}$ we have

$$
\operatorname{INVNC}(U)=\operatorname{INVNC}(\delta(U))+\operatorname{CDNC}(\delta(U)) .
$$

Proof Let $\lambda=\left(\lambda_{1}, \ldots, \lambda_{r}\right)$ be the type of $I$, and let $\pi$ be the path family in a $G$-tableau $U$, where $G=G_{\left[i_{1}, i_{1}+1\right]} \circ \cdots \circ G_{\left[i_{m}, i_{m}+1\right]}$.

Choose an index $j, 1 \leq j \leq m$ and let $\pi_{a}, \pi_{b}$ be the two paths which intersect at the central vertex of $G_{\left[i_{j}, i_{j}+1\right]}$, with $\pi_{b}$ entering from above, as in (4.2) or as in the first figure in (4.3). For some $k, \pi_{b}$ appears among the entries

$$
\lambda_{1}+\cdots+\lambda_{k-1}+1, \ldots, \lambda_{1}+\cdots+\lambda_{k}
$$

of $U$. The indices of these $\lambda_{k}$ paths increase from left to right in $U$, since the indices of all paths in $U$ form the permutation $u=u(I) \in \mathfrak{S}_{\lambda}^{-}(5.2)$.

Suppose that index $j$ is an inverted noncrossing of $U$ and therefore contributes 1 to $\operatorname{INVNC}(U)$. Then $\pi_{a}$ and $\pi_{b}$ intersect as in (4.2) and $\pi_{b}$ appears earlier than $\pi_{a}$ in $U$. If $\pi_{a}$ appears among the entries (5.6), then we must have $b<a$. Thus $\pi_{a}, \pi_{b}$ both appear in column $k$ of $\delta(U)$, and $j$ contributes 0 to $\operatorname{INVNC}(\delta(U))$ and 1 to $\operatorname{CDNC}(\delta(U))$. On the other hand, if $\pi_{a}$ does not appear in entries (5.6) of $U$, then it appears strictly to the right of column $k$ of $\delta(U)$. Thus $j$ contributes 1 to $\operatorname{INVNC}(\delta(U))$ and 0 to $\operatorname{CDNC}(\delta(U))$.

Now suppose that $j$ is not an inverted noncrossing in $U$ and therefore contributes 0 to $\operatorname{INVNC}(U)$. If $j$ is a crossing in $\pi$, then it contributes 0 to $\operatorname{INVNC}\left(\delta_{i}(U)\right)$ and $\operatorname{CDNC}(\delta(U))$. If $j$ is a noncrossing of $\pi$, then $\pi_{a}$ appears before $\pi_{b}$ in $U$. If $\pi_{a}$ appears before the entries (5.6) of $U$, then in $\delta(U)$ it appears in an earlier column than $\pi_{b}$ and contributes 0 to $\operatorname{INVNC}\left(\delta_{i}(U)\right)$ and $\operatorname{CDNC}(\delta(U))$. If $\pi_{a}$ appears as one of the entries (5.6) of $U$, then it appears in the same column of $\delta(U)$ as $\pi_{b}$ and satisfies $a<b$. Again $j$ contributes 0 to $\operatorname{INVNC}\left(\delta_{i}(U)\right)$ and $\operatorname{CDNC}(\delta(U))$.

Now we define the involution $\zeta=\zeta_{I}: \mathcal{T}_{I} \rightarrow \mathcal{T}_{I}$ as follows.

(1) If $W \in \mathcal{T}_{I}$ is column-strict, then define $\zeta(W)=W$.

(2) Otherwise,

(a) Let $t$ be the greatest index such that column $t$ of $W$ is not column-strict.

(b) Let $k$ be the greatest index such that two paths $\pi_{j}, \pi_{j^{\prime}}$ with $j, j^{\prime} \in I_{t}$ both pass through the central vertex of $G_{\left[i_{k}, i_{k+1}\right]}$, and let $\hat{\pi}=\left(\hat{\pi}_{1}, \ldots, \hat{\pi}_{n}\right)$ be the path family obtained from $\pi$ by swapping the terminal subpaths of $\pi_{j}$ and $\pi_{j^{\prime}}$, beginning at the central vertex of $G_{\left[i_{k}, i_{k+1}\right]} \cdot\left(\hat{\pi}_{i}=\pi_{i}\right.$ for $i \notin\left\{j, j^{\prime}\right\}$.)

(c) Define $\zeta(W)$ to be the tableau obtained from $W$ by replacing $\pi$ by $\hat{\pi}$.

Observe that each fixed point $W$ of $\zeta$ has type $e$ since it is column-closed and column-strict. On the other hand, when $W \in \mathcal{T}_{I}$ is not a fixed point of $\zeta$, one can show that the two tableaux $\delta^{-1}(W), \delta^{-1}(\zeta(W))$ in $\mathcal{U}_{I}$ are closely related. 
Lemma 5.2 Let $I=\left(I_{1}, \ldots I_{r}\right)$ be an ordered set partition of type $\lambda$, and define $u=u(I)$ as in (5.2). Let $G$-tableaux $W \in \mathcal{T}_{I}$ and $U, \widehat{U} \in \mathcal{U}_{I}$ satisfy $W=\delta(U) \neq$ $\zeta(W)=\delta(\widehat{U})$, and define path families $\pi, \hat{\pi}$ as above. Then for some generator $\in \in \mathfrak{S}_{\lambda}$ and some permutations $v, \hat{v}=s v \in \mathfrak{S}_{\lambda} u$ we have $U=U(\pi, u, v), \widehat{U}=U(\hat{\pi}, u, \hat{v})$.

Proof The tableaux $U, \widehat{U}$ contain the same path families as $W$ and $\zeta(W)$, respectively, and these path families are $\pi, \hat{\pi}$, as defined in the definition of $\zeta$.

Since elements of $\mathcal{T}_{I}$ are column-closed, it follows that the set of sink indices of paths in each column is equal to the set of source indices of paths in the same column. Thus the sequences $v, \hat{v}$ of sink indices, read bottom to top in columns $1, \ldots, r$, belong to $\mathfrak{S}_{\lambda} u$. By the definition of $\zeta$, the permutations $v$ and $\hat{v}$ differ from one another in exactly two positions: those holding the letters $v_{j}=\hat{v}_{j^{\prime}}$ and $v_{j^{\prime}}=\hat{v}_{j}$. Both letters belong to the same block of the ordered set partition $I$. Since we used the rightmost vertex in $\pi_{j} \cap \pi_{j^{\prime}}$ to define $\hat{\pi}_{j}$ and $\hat{\pi}_{j^{\prime}}$, the letters must appear consecutively in $v$ and in $\hat{v}$. It follows that $\hat{v}=s v$ for some adjacent transposition $s \in \mathfrak{S}_{\lambda}$.

Furthermore, when $W \in \mathcal{T}_{I}$ is not a fixed point of $\zeta$, the values of the statistics INVNC and CDNC on $W$ and $\zeta(W)$ are closely related.

Proposition 5.3 Let $W \in \mathcal{T}_{I}$ satisfy $W=\delta(U(\pi, u, v)) \neq \zeta(W)=\delta(U(\hat{\pi}, u, \hat{v}))$ for $\pi, \hat{\pi}, v, \hat{v}$ as in Lemma 5.2. Then we have

$$
\begin{aligned}
& \operatorname{INVNC}(\zeta(W))=\operatorname{INVNC}(W), \\
& \operatorname{CDNC}(\zeta(W))+\frac{\mathrm{C}(\hat{\pi})}{2}= \begin{cases}\operatorname{CDNC}(W)+\frac{\mathrm{C}(\pi)+1}{2} & \text { if } v<\hat{v} \\
\operatorname{CDNC}(W)+\frac{\mathrm{C}(\pi)-1}{2} & \text { if } v>\hat{v}\end{cases}
\end{aligned}
$$

Proof To verify (5.7), let $t, k$ be as in the definition of $\zeta$ and let $\ell$ be an index which does not belong to $I_{t}$. Then $\pi_{\ell}$ is an entry of $W$ and $\zeta(W)$ which does not appear in column $t$. Clearly, any point of intersection between $\pi_{\ell}$ and $\pi_{j}$ or $\pi_{j^{\prime}}$ which occurs in $G$ to the left of the central vertex of $G_{\left[i_{k}, i_{k}+1\right]}$ exactly matches an intersection between $\pi_{\ell}$ and $\hat{\pi}_{j}$ or $\hat{\pi}_{j^{\prime}}$. On the other hand, suppose that $\pi_{\ell}$ and $\pi_{j}$ (or $\pi_{j^{\prime}}$ ) have a point of intersection in $G$ to the right of the central vertex of $G_{\left[i_{k}, i_{k}+1\right]}$. Since $j$ and $j^{\prime}$ both belong to $I_{t}$, this point is an inverted noncrossing of $\pi_{\ell}$ and $\pi_{j}$ (or $\pi_{j^{\prime}}$ ) in $W$ if and only if it is an inverted noncrossing of $\pi_{\ell}$ and $\hat{\pi}_{j^{\prime}}\left(\right.$ or $\left.\hat{\pi}_{j}\right)$ in $\zeta(W)$.

To verify (5.7), consider the intersection of $\pi_{j}$ and $\pi_{j^{\prime}}$ at the central vertex of $G_{\left[i_{k}, i_{k}+1\right]}$ and the tableaux $U(\pi, u, v), U(\hat{\pi}, u, \hat{v})$. If $v>\hat{v}$, then our choice of $\left(j, j^{\prime}\right)$ implies that this intersection is either a defective noncrossing or a crossing that sends $\pi_{j}$ above $\pi_{j^{\prime}}$. In the first case, the map $\zeta$ removes exactly one defective noncrossing and creates exactly one crossing, changing the statistic sum CDNC $+\mathrm{C} / 2$ by $-1+1 / 2=$ $-1 / 2$. In the second case, the map $\zeta$ removes exactly one crossing and creates exactly one proper noncrossing, changing the same sum by $-1 / 2+0=-1 / 2$. Similarly, if $v<\hat{v}$ then our choice of $\left(j, j^{\prime}\right)$ implies that the intersection is either a proper noncrossing or a crossing that sends $\pi_{j^{\prime}}$ above $\pi_{j}$. In both cases, the statistic sum increases by $1 / 2$.

Finally we can state and justify a subtraction-free formula for $\epsilon_{q}^{\lambda}\left(\left(1+T_{s_{i_{1}}}\right) \cdots\left(1+T_{s_{i_{m}}}\right)\right)$. 
Theorem 5.4 Let $G$ be the wiring diagram of $s_{i_{1}} \cdots s_{i_{m}}$. Then for $\lambda \vdash n$ we have

$$
\epsilon_{q}^{\lambda}\left(\left(1+T_{s_{i_{1}}}\right) \cdots\left(1+T_{s_{i_{m}}}\right)\right)=\sum_{W} q^{\mathrm{INVNC}(W)+\mathrm{C}(W) / 2}
$$

where the sum is over all column-strict G-tableaux of type e and shape $\lambda^{\top}$.

Proof Let $B$ be the path matrix of $G$. Combining Theorems 3.7 and [9, Thm.5.4] (i.e., (5.1)) with the identity (5.3), we see that the left-hand side of (5.9) is

$$
\begin{aligned}
\sigma_{B}\left(\operatorname{Imm}_{\epsilon_{q}^{\lambda}}(x)\right) & =\sum_{I} \sigma_{B}\left(\operatorname{det}_{q}\left(x_{I_{1}, I_{1}}\right) \cdots \operatorname{det}_{q}\left(x_{I_{r}, I_{r}}\right)\right) \\
& =\sum_{I} \sum_{y \in \mathfrak{S}_{\lambda}}(-1)^{\ell(y)} q_{y}^{-1} \sigma_{B}\left(x^{u(I), y u(I)}\right),
\end{aligned}
$$

where the first two sums are over ordered set partitions $I=\left(I_{1}, \ldots, I_{r}\right)$ of $[n]$ of type $\lambda$. Fixing one such partition $I$ and writing $u=u(I)$, we may use Proposition 4.1 and Lemma 5.1 to express the third sum as

$$
\sum_{y \in \mathfrak{S}_{\lambda}} \sum_{\pi}(-1)^{\ell(y)} q_{y}^{-1} q^{\frac{\mathrm{C}(\pi)}{2}} q^{\mathrm{INVNC}(U(\pi, u, y u))}=\sum_{y \in \mathfrak{S}_{\lambda}} \sum_{\pi}(-1)^{\ell(y)} q_{y}^{-1} q^{\frac{\mathrm{C}(\pi)}{2}} q^{\mathrm{INVNC}(W)+\operatorname{CDNC}(W)},
$$

where the inner sums are over path families $\pi$ of type $u^{-1} y u$ which cover $G$, and where $W=\delta_{I}(U(\pi, u, y u))$. As $y$ and $\pi$ vary in the above sums, $U(\pi, u, y u)$ varies over all tableaux in $\mathcal{U}_{I}$, and $W$ varies over all tableaux in $\mathcal{T}_{I}$.

Now consider a tableau $W \in \mathcal{T}_{I}$ which satisfies $\zeta(W) \neq W$. Let tableaux $W$ and $\zeta(W)$ contain path families $\pi$ of type $u^{-1} y u$ and $\hat{\pi}$ of type $u^{-1} \hat{y} u$, respectively. By Lemma 5.2 we have $\hat{y}=s y$ for some $s \in \mathfrak{S}_{\lambda}$. Assume without loss of generality that $y<\hat{y}$ and consider the term on the right-hand side of (5.11) corresponding to $\zeta(W)$,

$$
(-1)^{\ell(\hat{y})} q_{y^{\prime}}^{-1} q^{\frac{\mathrm{C}(\hat{\pi})}{2}} q^{\operatorname{INVNC}(\zeta(W))+\operatorname{CDNC}(\zeta(W))} .
$$

By Proposition 5.3, this is

$$
(-1)^{\ell(y)+1} q_{y}^{-1} q^{-\frac{1}{2}} q^{\frac{\mathrm{C}(\pi)+1}{2}} q^{\mathrm{INVNC}(W)+\mathrm{CDNC}(W)}=-(-1)^{\ell(y)} q_{y}^{-1} q^{\frac{\mathrm{C}(\pi)}{2}} q^{\mathrm{INVNC}(W)+\mathrm{CDNC}(W)}
$$

i.e., the opposite of the term corresponding to $W$. Thus it suffices to sum the righthand side of (5.11) over only the pairs $(y, \pi)$ corresponding to tableaux $W$ satisfying $W=\zeta(W)$. By the definition of $\zeta$, each such tableau $W$ is column-strict and therefore satisfies $\operatorname{CDNC}(W)=0$. By the definition of $\mathcal{T}_{I}$, each such tableau has type $e$. Thus each tableau

$$
\delta_{I}^{-1}(W)=U=U(\pi, u, y u) \in \mathcal{U}_{I}
$$


satisfies $y=\operatorname{type}(\pi)=e$. It follows that the right-hand side of (5.11) and the third sum in (5.10) are equal to

$$
\sum_{W} q^{\frac{\mathrm{C}(\pi)}{2}} q^{\mathrm{INVNC}(W)}
$$

where the sum is over all tableau $W$ in $\mathcal{T}_{I}$ which are column-strict of type $e$. By the definition of $\mathcal{T}_{I}$, each such tableau has shape $\lambda^{\top}$. Thus the three expressions in (5.10) are equal to the right-hand side of (5.9).

To illustrate the theorem, we compute $\epsilon_{q}^{21}\left(\left(1+T_{s_{1}}\right)\left(1+T_{S_{2}}\right)\left(1+T_{s_{1}}\right)\right)$ using the wiring diagram (3.11). There are two path families of type $e$ which cover $G$, and one column-strict $G$-tableau of shape $21^{\top}=21$ for each:

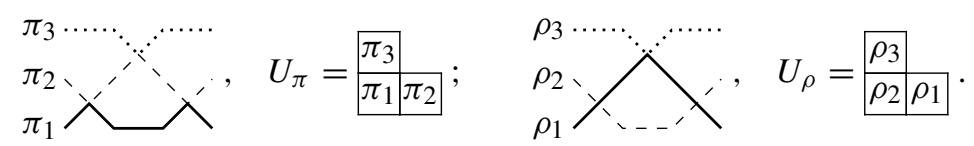

Tableau $U_{\pi}$ contributes $q^{\mathrm{INVNC}\left(U_{\pi}\right)} q^{\mathrm{C}\left(U_{\pi}\right) / 2}=q^{1} q^{0 / 2}=q$, since $\pi$ has no crossings, and for only one of its noncrossings are the two paths inverted in $U_{\pi}: \pi_{3}$ intersects $\pi_{2}$ from above and appears in an earlier column of $U_{\pi}$. Tableau $U_{\rho}$ contributes $q^{\mathrm{INVNC}\left(U_{\rho}\right)} q^{\mathrm{C}\left(U_{\rho}\right) / 2}=q^{1} q^{2 / 2}=q^{2}$, since $\rho$ has two crossings, and for its unique noncrossing, the two paths are inverted in $U_{\rho}: \rho_{3}$ intersects $\rho_{1}$ from above and appears in an earlier column of $U_{\rho}$. Adding the two contributions together, we have $\epsilon_{q}^{21}\left(\left(1+T_{s_{1}}\right)\left(1+T_{s_{2}}\right)\left(1+T_{s_{1}}\right)\right)=q+q^{2}$.

Using the other reduced expression $s_{2} s_{1} s_{2}$ for the long element of $\mathfrak{S}_{3}$, one similarly computes $\epsilon_{q}^{21}\left(\left(1+T_{s_{2}}\right)\left(1+T_{s_{1}}\right)\left(1+T_{s_{2}}\right)\right)=q+q^{2}$. It is not generally true, however, that distinct reduced expressions $s_{i_{1}} \cdots s_{i_{m}}$ and $s_{j_{1}} \cdots s_{j_{m}}$ for $w \in \mathfrak{S}_{n}$ lead to equal evaluations $\epsilon_{q}^{\lambda}\left(\left(1+T_{s_{i_{1}}}\right) \cdots\left(1+T_{s_{i_{\ell}}}\right)\right)$ and $\epsilon_{q}^{\lambda}\left(\left(1+T_{s_{j_{1}}}\right) \cdots\left(1+T_{s_{j_{\ell}}}\right)\right)$. For example, consider the reduced expressions $s_{3} s_{2} s_{1} s_{2}, s_{3} s_{1} s_{2} s_{1}$ for $3241 \in \mathfrak{S}_{4}$, the corresponding wiring diagrams $G, H$, and the $G$ - and $H$-tableaux of type $e$ and shape 211. It is easy to see that there is only one column-strict $G$-tableau of this type and shape, while there are no such column-strict $H$-tableaux. Since the $G$-tableau has one inverted noncrossing and two crossings, we have

$$
\begin{aligned}
& \epsilon_{q}^{31}\left(\left(1+T_{s_{3}}\right)\left(1+T_{s_{2}}\right)\left(1+T_{s_{1}}\right)\left(1+T_{s_{2}}\right)\right)=q^{2} \\
& \epsilon_{q}^{31}\left(\left(1+T_{s_{3}}\right)\left(1+T_{s_{1}}\right)\left(1+T_{s_{2}}\right)\left(1+T_{s_{1}}\right)\right)=0
\end{aligned}
$$

A special case of Theorem 5.4 allows one to combinatorially interpret evaluations of $\epsilon_{q}^{\lambda}$ at certain elements $q_{w} C_{w}^{\prime}(q)$ of the Kazhdan-Lusztig basis of $H_{n}(q)$. Call a permutation 321-hexagon-avoiding if it avoids the patterns 321, 56781234, 56718234, $46781235,46718235$. 
Corollary 5.5 Let $G$ be the wiring diagram of a reduced expression $s_{i_{1}} \cdots s_{i_{m}}$ for a 321-hexagon-avoiding permutation $w \in \mathfrak{S}_{n}$. Then we have

$$
\epsilon_{q}^{\lambda}\left(q_{w} C_{w}^{\prime}(q)\right)=\sum_{U} q^{\mathrm{INVNC}(U)+\mathrm{C}(U) / 2}
$$

where the sum is over all column-strict G-tableaux of type e and shape $\lambda^{\top}$.

Proof Billey and Warrington [2, Thm.1] showed that for every reduced expression $s_{i_{1}} \cdots s_{i_{m}}$ of a 321-hexagon-avoiding permutation $w$, we have $q_{w} C_{w}^{\prime}(q)=$ $\left(1+T_{s_{i_{1}}}\right) \cdots\left(1+T_{s_{i_{m}}}\right)$.

A subclass of the 321-hexagon-avoiding permutations is the set of permutations avoiding the patterns 321 and 3412. The special case of Corollary 5.5 corresponding to these permutations is equivalent to the special case of [4, Thm.6.4] corresponding to these permutations. Given a column-strict $G$-tableau $U$ containing a path family $\pi=\left(\pi_{1}, \ldots, \pi_{n}\right)$, define $\operatorname{INV}(U)$ to be the number of intersecting pairs $\left(\pi_{i}, \pi_{j}\right)$ with $j>i$ and $\pi_{j}$ appearing in an earlier column of $U$ than $\pi_{i}$.

Corollary 5.6 Let $G$ be the wiring diagram of a reduced expression $s_{i_{1}} \cdots s_{i_{m}}$ for a 321-avoiding, 3412-avoiding permutation $w \in \mathfrak{S}_{n}$. Then we have

$$
\epsilon_{q}^{\lambda}\left(q_{w} C_{w}^{\prime}(q)\right)=\sum_{U} q^{\mathrm{INV}(U)}
$$

where the sum is over all column-strict G-tableaux of type e and shape $\lambda^{\top}$.

Proof Since $w$ is 321-hexagon-avoiding, we have the formula (5.12). Since $w$ also avoids the pattern 3412, the results [12, Thm.4.3, Prop. 4.4] imply that $G$ has the structure of a zig-zag network [12, Sec.5]. Thus the unique family $\pi=\left(\pi_{1}, \ldots, \pi_{n}\right)$ which covers $G$ and has type $e$ satisfies

(1) $\pi$ has no crossings,

(2) the intersection of any two paths of $\pi$ is either empty, or consists of a single connected component.

Thus we have that $\mathrm{C}(U)=0$ for each tableau $U$ in (5.12). Since $\pi$ covers $G$, we also have that each nonempty intersection $\pi_{i} \cap \pi_{j}$ is a single vertex, more specifically a noncrossing. It follows that each tableau $U$ in (5.12) satisfies $\operatorname{INVNC}(U)=\operatorname{INV}(U)$.

\section{Open problems}

While Theorem 5.4 provides a method for evaluating all $H_{n}(q)$-characters at all elements $\left(1+T_{s_{i_{1}}}\right) \cdots\left(1+T_{s_{i_{m}}}\right)$ of $H_{n}(q)$, this method leads to formulas involving subtraction: not all $H_{n}(q)$-characters belong to the $\mathbb{N}[q]$-span of the induced sign characters $\left\{\epsilon_{q}^{\lambda} \mid \lambda \vdash n\right\}$. It would therefore be interesting to state and prove an analog of Theorem 5.4 for irreducible characters, since all other $H_{n}(q)$-characters belong to 
the $\mathbb{N}[q]$-span of these [see the table following Eq. (1.4)]. This would also provide a combinatorial proof of a weakening of Haiman's result [6, Lem. 1.1].

Problem 6.1 For all irreducible characters $\chi_{q}^{\lambda}$ and all sequences $\left(s_{i_{1}}, \ldots s_{i_{m}}\right)$ of generators of $\mathfrak{S}_{n}$, combinatorially interpret the evaluation $\chi_{q}^{\lambda}\left(\left(1+T_{s_{i_{1}}}\right) \cdots\left(1+T_{s_{i_{m}}}\right)\right)$.

Another possibility of strengthening Theorem 5.4 concerns Kazhdan-Lusztig basis elements and parabolic subgroups of $\mathfrak{S}_{n}$. For $1 \leq a \leq b \leq n$, let $s_{[a, b]}$ denote the longest element of the parabolic subgroup $\mathfrak{S}_{[a, b]}$ of $\mathfrak{S}_{n}$ generated by $s_{a}, \ldots, s_{b-1}$. Sometimes called a reversal, $s_{[a, b]}$ has one-line notation

$$
1 \cdots(a-1) \cdot b(b-1) \cdots a \cdot(b+1) \cdots n
$$

and the corresponding modified Kazhdan-Lusztig basis element satisfies

$$
q_{s_{[a, b]}} C_{S_{[a, b]}}^{\prime}(q)=\sum_{w \in \mathfrak{S}_{[a, b]}} T_{w} .
$$

In the special case that $b=a+1$, this is $q_{s_{a}} C_{s_{a}}^{\prime}(q)=1+T_{s_{a}}$.

For some permutations $w \in \mathfrak{S}_{n}$, there exists a polynomial $g_{w}(q) \in \mathbb{N}[q]$ and a reversal factorization $w=s_{\left[c_{1}, d_{1}\right]} \cdots s_{\left[c_{m}, d_{m}\right]}$ such that the modified Kazhdan-Lusztig basis element $q_{w} C_{w}^{\prime}(q)$ satisfies

$$
g(q) q_{w} C_{w}^{\prime}(q)=q_{s_{\left[c_{1}, d_{1}\right]}} C_{S_{\left[c_{1}, d_{1}\right]}}^{\prime}(q) \cdots q_{s_{\left[c_{m}, d_{m}\right]}} C_{s_{\left[c_{m}, d_{m}\right]}^{\prime}}^{\prime}(q)
$$

No characterization of these permutations is known, but two sets of sufficient conditions are 321-hexagon avoidance [2, Thm.1] and 3412-, 4231-avoidance [12, Thm.4.3]. Work of Stembridge [16, Sec.5] suggests that 45312-avoidance may be a necessary condition. In order to improve our ability to evaluate $H_{n}(q)$-characters at Kazhdan-Lusztig basis elements, it would therefore be interesting to solve the following problem.

Problem 6.2 For all characters $\theta_{q} \in\left\{\epsilon_{q}^{\lambda} \mid \lambda \vdash n\right\}$ or $\theta_{q} \in\left\{\chi_{q}^{\lambda} \mid \lambda \vdash n\right\}$, and all sequences $\left(s_{\left[c_{1}, d_{1}\right]}, \ldots, s_{\left[c_{m}, d_{m}\right]}\right)$ of reversals in $\mathfrak{S}_{n}$, combinatorially interpret the evaluation

$$
\theta_{q}\left(q_{s_{\left[c_{1}, d_{1}\right]}} C_{s_{\left[c_{1}, d_{1}\right]}^{\prime}}^{\prime}(q) \cdots q_{s_{\left[c_{m}, d_{m}\right]}} C_{s_{\left[c_{m}, d_{m}\right]}^{\prime}}^{\prime}(q)\right)
$$

For $\theta_{q}$ varying over $\left\{\epsilon_{q}^{\lambda} \mid \lambda \vdash n\right\}$, this would provide a common generalization of Theorem 5.4 and [4, Thm. 6.4]. For $\theta_{q}$ varying over $\left\{\chi_{q}^{\lambda} \mid \lambda \vdash n\right\}$, this would provide a common generalization of the solution to Problem 6.1 and [4, Thm. 8.1] and would provide a combinatorial interpretation of special cases of [6, Lem. 1.1]. Furthermore, by [15, Cor. 3.3] we have that for each $n \times n$ totally nonnegative matrix $B$, and each partition $\lambda \vdash n$, the evaluation $\operatorname{Imm}_{\chi^{\lambda}}(B)$ is nonnegative. While this evaluation has no known combinatorial interpretation, it is known to be equal to a nonnegative linear 
combination of evaluations of the form $\chi^{\lambda}\left(\left(C_{S_{\left[c_{1}, d_{1}\right]}^{\prime}}^{\prime}(1) \cdots C_{S_{\left[c_{m}, d_{m}\right]}^{\prime}}^{\prime}(1)\right)\right.$. Thus a solution to the above problem would provide the desired combinatorial interpretation of $\operatorname{Imm}_{\chi^{\lambda}}(B)$.

\section{References}

1. Beck, D., Remmel, J., Whitehead, T.: The combinatorics of transition matrices between the bases of the symmetric functions and the $B_{n}$ analogues. Discrete Math. 153, 3-27 (1996)

2. Billey, S.C., Warrington, G.: Kazhdan-Lusztig polynomials for 321-hexagon-avoiding permutations. J. Algebr. Combin. 13(2), 111-136 (2001)

3. Björner, A., Brenti, F.: Combinatorics of Coxeter Groups, Graduate Texts in Mathmatics, vol. 231. Springer, New York (2005)

4. Clearman, S., Hyatt, M., Shelton, B., Skandera, M.: Evaluations of Hecke algebra traces at KazhdanLusztig basis elements. Electron. J. Combin. 23, 2 (2016)

5. Deodhar, V.: A combinatorial settting for questions in Kazhdan-Lusztig theory. Geom. Dedicata 36(1), 95-119 (1990)

6. Haiman, M.: Hecke algebra characters and immanant conjectures. J. Am. Math. Soc. 6(3), 569-595 (1993)

7. Karlin, S., McGregor, G.: Coincidence probabilities. Pac. J. Math. 9, 1141-1164 (1959)

8. Kazhdan, D., Lusztig, G.: Representations of Coxeter groups and Hecke algebras. Invent. Math. 53, 165-184 (1979)

9. Konvalinka, M., Skandera, M.: Generating functions for Hecke algebra characters. Can. J. Math. 63(2), 413-435 (2011)

10. Lindström, B.: On the vector representations of induced matroids. Bull. Lond. Math. Soc. 5, 85-90 (1973)

11. Ram, A.: A Frobenius formula for the characters of the Hecke algebras. Invent. Math. 106(3), 461-488 (1991)

12. Skandera, M.: On the dual canonical and Kazhdan-Lusztig bases and 3412-, 4231-avoiding permutations. J. Pure Appl. Algebra 212, 1086-1104 (2008)

13. Stanley, R.: Enumerative Combinatorics, vol. 2. Cambridge University Press, Cambridge (1999)

14. Stanley, R.: Positivity problems and conjectures. In: Arnold, V., Atiyah, M., Lax, P., Mazur, B. (eds.) Mathematics: Frontiers and Perspectives, pp. 295-319. American Mathematical Society, Providence (2000)

15. Stembridge, J.: Immanants of totally positive matrices are nonnegative. Bull. Lond. Math. Soc. 23, 422-428 (1991)

16. Stembridge, J.: Some conjectures for immanants. Can. J. Math. 44(5), 1079-1099 (1992)

17. Zhang, R.B.: Howe duality and the quantum general linear group. Proc. Am. Math. Soc. 131(9), 2681-2692 (2003) 